Pacific Journal of Mathematics

ON SIMPLE EXTENDED LIE ALGEBRAS OVER FIELDS O 


\title{
ON SIMPLE EXTENDED LIE ALGEBRAS OVER FIELDS OF CHARACTERISTIC ZERO
}

\author{
ARThur A. SAgLE
}

In this paper we shall investigate algebras which generalize Lie algebras, Malcev algebras and binary-Lie algebras (every two elements generate a Lie subalgebra). Such an algebra $A$ is called an extented Lie algebra (briefly el-algebra) and is defined by

$$
x y=-y x \quad \text { and } \quad J(x, y, x y)=0
$$

for all $x, y$ in $A$ where $J(x, y, z)=x y \cdot z+y z \cdot x+z x \cdot y$. We prove the following.

Theorem. Let $A$ be a simple finite dimensional el-algebra over an algebraically closed field of characteristic zero, then $A$ is a simple Lie algebra or the simple seven dimensional Malcev algebra if and only if the trace form, $(x, y)=$ trace $R_{x} R_{y}$, is a nondegenerate invariant form.

The identities for right multiplications in the Lie [1] and Malcev [2] algebras mentioned in the theorem yield that the form $(x, y)$ is nondegenerate and invariant i.e. $(x y, z)=(x, y z)$; so this paper is concerned with the converse statement. All algeras considered in this paper are finite dimensional.

2. Precartan subalgebras. In this section we shall consider subalgebras of an arbitrary anti-commutative algebra analogous to Cartan subalgebras of a Lie algebra.

Definition. A subalgebra $N$ of an anti-commutative algebra $A$ is a precartan subalgebra if

1. $\quad N$ is a nilpotent Lie subalgebra of $A$;

2. the mapping $N \rightarrow E_{L}(A): n \rightarrow R_{n}$ is a representation of $N$ where $R_{x}$ or $R(x)$ denotes the mapping $a \rightarrow a x$ and $E_{L}(A)$ denotes the Lie algebra of all linear transformations on $A$.

Thus $N$ is a Lie subalgebra of $A$ such that there exists an integer $k$ with $\left(\cdots\left(n_{1} n_{2}\right) \cdots n_{k}\right)=0$ for all $n_{i}$ in $N$ and $\left[R_{n}, R_{m}\right]=R_{n m}$ for all $n, m$ in $N$.

Now $R(N)=\left\{R_{n}: n \in N\right\}$ is a Lie algebra of linear transformations which is also a nilpotent Lie algebra of linear transformations: GP-1453.

Received November 7, 1963. This research was sponsored in part by NSF Grant 


$$
\begin{aligned}
\left.\left.0=R\left[\left(n_{1} n_{2}\right) \cdots\right) n_{k}\right)\right] & \left.=\left[R\left(\left(n_{1} n_{2}\right) \cdots\right) n_{k-1}\right), R\left(n_{k}\right)\right] \\
& =\cdots=\left[\cdots\left[R\left(n_{1}\right), R\left(n_{2}\right)\right], \cdots, R\left(n_{k}\right)\right] .
\end{aligned}
$$

So if we assume the base field $F$ is algebraically closed we can, analogous to Lie algebra theory, decompose $A$ into a direct sum of weight spaces relative to $R(N)$ :

$$
A=A(N, 0) \oplus \sum_{\alpha \neq 0} A(N, \alpha)
$$

where if $\lambda: R(N) \rightarrow F: R_{n} \rightarrow \lambda\left(R_{n}\right) \equiv \lambda(n)$ is a weight of $R(N)$, then the weight space corresponding to $\lambda$ is

$$
\begin{aligned}
& A(N, \lambda)=\left\{x \text { in } A: \text { all } n \text { in } N, x\left(R_{n}-\lambda(n) I\right)^{t}=0\right. \\
& \quad \text { for some integer } t>0\}=\bigcap_{n \in N} A\left(R_{n}, \lambda(n)\right)
\end{aligned}
$$

where $A\left(R_{n}, \lambda(n)\right)=\left\{x \in A: x\left(R_{n}-\lambda(n) I\right)^{m}=0\right.$ for some integer $\left.m>0\right\}$. By Lie's theorem the weights $\lambda$ are linear functionals on $R(N)$ and can actually be considered as linear functionals on $N$ via $\lambda(n) \equiv \lambda\left(R_{n}\right)$ and noting $R_{n}$ is a linear transformation. The following facts concerning the above decomposition are known from Lie algebra theory [1].

THEOREM 2.1.

1. Precartan subalgebras exist.

2. The weight spaces $A(N, \lambda)$ are $R(N)$-invariant subspaces.

3. $\lambda$ is the only weight of $R(N)$ in $A(N, \lambda)$.

4. If $M$ is a precartan subalgebra of $A$ containing $N$, then $M \subset A(N, 0)$.

5. There exists an element $n$ in $N$ such that $A(N, 0)=A\left(R_{n}, 0\right)$.

6. There are finitely many weights.

Definition. The normalizer $Z(B)$ of a subalgebra $B$ of an anticommutative algebra $A$ is the set of all $x$ in $A$ such that $x B \subset B$.

Proposition 2.2. Let $N$ be a precartan subalgebra of an anticommutative algebra $A$ over an algebraically closed field, then $N=$ $Z(N)$ if and only if $N=A(N, 0)$.

Proof. First note $Z(N) \subset A(N, 0)$. For if $x \in Z(N)$, then for all $n \in N, x R_{n}=x n \in N$ and since $N$ is a nilpotent Lie subalgebra of $A$, there exists an integer $k$ with $x R_{n}^{k}=0$ and so $x \in A(N, 0)$. Thus noting that $N \subset Z(N)$ we always have

$$
N \subset Z(N) \subset A(N, 0) .
$$

In particular if $N \neq Z(N)$, then $N \neq A(N, 0)$.

Next assume $N \neq A(N, 0)$, then we shall show $N \neq Z(N) . \quad A(N, 0)$ and $N$ are $R(N)$-invariant subspaces and $R(N)$ restricted to $A(N, 0)$ is 
a nilpotent Lie algebra of linear transformations. Hence we obtain a nilpotent Lie algebra of linear transformations $\overline{R(N)}$ acting in the nonzero space $A(N, 0) / N$. Now by Engel's theorem there exists an $\bar{x}=x+N \neq \overline{0}$ in $A(N, 0) / N$ such that $\overline{x R(N)}=\overline{0}$, But this means $\overline{0}=\bar{x} \bar{R}_{n}=\overline{x R}_{n}$ for all $n$ in $N$ and so $x N \subset N$. But by definition of $Z(N), x \in Z(N)$. However $\bar{x} \neq 0$ means $x$ is not in $N$ and so $N \neq Z(N)$.

Definition. A precartan subalgebra $N$ of an anti-commutative algebra $A$ is a Cartan subalgebra of $A$ if $N=Z(N)$.

Cartan subalgebras are difficult to find, however if there exists an element $u$ in $A$ such that $N=A\left(R_{u}, 0\right)$ is a precartan subalgebra of $A$ over an algebraically closed field, then $N$ is a Cartan subalgebra. For decompose $A$ relative to $R(N)$, then $N \subset A(N, 0)=\cap_{n \in N} A\left(R_{n}, 0\right) \subset$ $A\left(R_{u}, 0\right)=N$ and the results follow from Proposition 2.2.

The following notation will be used: if $h\left(x_{1}, \cdots, x_{n}\right)$ is a function of $n$ indeterminates such that for any $n$ subsets $B_{i}$ of $A$ the elements $h\left(b_{1}, \cdots, b_{n}\right)$, for $b_{i} \in B_{i}$, are in $A$, then $h\left(B_{1}, \cdots, B_{n}\right)$ denotes the linear subspace spanned by all the elements $h\left(b_{1}, \cdots, b_{n}\right)$ for $b_{i} \in B_{i}$. Also we shall identity the element $b$ and the set $\{b\}$.

3. Identities. We shall now restrict ourselves to el-algebras, that is, we assume the algebra $A$ satisfies

$$
x y=-y x \quad \text { and } \quad J(x, y, x y)=0
$$

for all $x, y$ in $A$. First we note for any anti-commutative algebra $A$ that a straightforward calculation yields

$$
\begin{aligned}
w J(x, y, z) & -x J(y, z, w)+y J(z, w, x)-z J(w, x, y) \\
& =J(w x, y, z)+J(y z, w, x) \\
& +J(w y, z, x)+J(z x, w, y) \\
& +J(w z, x, y)+J(x y, w, z)
\end{aligned}
$$

for all $w, x, y, z$ in $A$.

Next a linearization of (3.1) yields

$$
J(x y, y, z)+J(z y, y, x)=0
$$

for all $x, y, z$ in $A$ and a further linearization of (3.3) yields

$$
\begin{aligned}
J(w x, y, z)+J(y z, w, x) & =J(w y, z, x)+J(z x, w, y) \\
& =J(w z, x, y)+J(x y, w, z)
\end{aligned}
$$

for all $w, x, y, z$ in A. Combining (3.2) and (3.4) we have

$$
\begin{aligned}
w J(x, y, z) & -x J(y, z, w)+y J(z, w, x)-z J(w, x, y) \\
& =3[J(w x, y, z)+J(y z, w, x)]
\end{aligned}
$$


for all $w, x, y, z$ in $A$.

For the remainder of this paper we shall assume $A$ is a simple, finite dimensional el-algebra over an algebraically closed field of characteristic zero; however it should be clear when these various conditions can be relaxed. Let $N$ be a precartan subalgebra of A and decompose

$$
A=A(N, 0) \oplus \sum_{\lambda \neq 0} A(N, \lambda)
$$

relative to $R(N)$. Next let $m, n \in N$ and $w, z \in A$, then from (3.4) we have

$$
\begin{aligned}
J(w n, m, z)+J(m z, w, n) & =J(w m, z, n)+J(z n, w, m) \\
& =J(w z, n, m)+J(n m, w, z) \\
& =J(n m, w, z)
\end{aligned}
$$

since $m, n \in N$ and $J(A, n, m)=0$. Now set $w_{1}=w R_{n}$ and $w_{k}$ $=w_{k-1} R_{n}=w R_{n}^{k}$ and set $z_{1}=z R_{m}, z_{k}=z_{k-1} R_{n}+z R\left(m R_{n}^{k-1}\right)$ for $k=2,3, \cdots$. From the above set of equations we have

$$
J\left(w R_{n}, m, z\right)=J\left(w, m R_{n}, z\right)+J\left(w, n, z R_{m}\right),
$$

that is,

$$
J\left(w_{1}, m, z\right)=J\left(w, m R_{n}, z\right)+J\left(w, n, z_{1}\right) .
$$

Now assume

$$
J\left(w_{k}, m, z\right)=J\left(w, m R_{n}^{k}, z\right)+J\left(w, n, z_{k}\right),
$$

then for $k+1$ we have

$$
\begin{aligned}
J\left(w_{k+1}, m, z\right) & =J\left(v R_{n}^{k}, m, z\right), \quad \text { where } v=w R_{n} \\
& =J\left(v_{k}, m, z\right), \quad \text { where } v_{k}=v R_{n}^{k} \\
& =J\left(v, m R_{n}^{k}, z\right)+J\left(v, n, z_{k}\right), \text { using induction hypothesis } \\
& =J\left(w R_{n}, m R_{n}^{k}, z\right)+J\left(w R_{n}, n, z_{k}\right) \\
& =J\left(w,\left(m R_{n}^{k}\right) R_{n}, z\right)+J\left(w, n, z R\left(m R_{n}^{k}\right)\right) \\
& +J\left(w, n R_{n}, z_{k}\right)+J\left(w, n, z_{k} R_{n}\right), \quad \text { using (3.6) twice } \\
& =J\left(w, m R_{n}^{k+1}, z\right)+J\left(w, n, z_{k+1}\right) .
\end{aligned}
$$

Thus for every $m, n \in N$ and $w, z \in A$,

$$
J\left(w R_{n}^{k}, m, z\right)=J\left(w, m R_{n}^{k}, z\right)+J\left(w, n, z_{k}\right)
$$

where $z_{1}=z R_{n}$ and $z_{k}=z_{k-1} R_{n}+z R\left(m R_{n}^{k}\right) k=2,3, \cdots$.

Let $l$ be the dimension of $A(N, 0)$, then choosing $k$ large enough e.g. $l \geqq l+2$ and using $N \subset A(N, 0)$ we have $m R_{n}^{k}=0$ and

$$
J\left(w_{k}, m, z\right)=J\left(w, n, z_{k}\right)
$$

where $k \geqq l+2, w_{k}=w R_{n}^{k}$ and now $z_{k}=z_{k-1} R_{n}$. 
We shall use (3.8) with the following lemmas to prove

$$
A(N, \sigma) A(N, \rho) \subset A(N, \rho+\sigma) \text { if } \rho \neq \sigma .
$$

LEMmA 3.10. Let $\rho$ be any weight of $N$ and $n \in N$, then $R_{n}$ is nonsingular on $A(N, \rho)$ if and only if $\rho(n) \neq 0$.

LEMMA 3.11. Let $\rho, \sigma$ be any nonzero weights of $N$ in $A$ with $\rho \neq \sigma$, then there exists $u \in N$ such that $\rho(u) \neq \sigma(u)$ and $R_{u}$ is nonsingular on $A(N, \rho)$.

Proof. Since $\rho \neq \sigma$, there exists $h \in N$ with $\rho(h) \neq \sigma(h)$. If $R_{h}$ is nonsingular on $A(N, \rho)$, we are finished. Otherwise $R_{h}$ is singular on $A(N, \rho)$ and by Lemma 3.10, $0=\rho(h) \neq \sigma(h)$. Now there exists $k \in N$ so that $R_{k}$ is nonsingular on $A(N, \rho)$, since $\rho \neq 0$ and so $\rho(k) \neq 0$. If $\sigma(k) \neq \rho(k)$, then we are finished. Otherwise if $\sigma(k)=\rho(k)$, set $u=h+k$ and note $\sigma(u)=\sigma(h)+\sigma(k)=\sigma(h)+\rho(k) \neq \rho(k)=\rho(k)+$ $\rho(h)=\rho(h+k)=\rho(u)$ and also $\rho(u) \neq 0$ so that $R_{u}$ is nonsingular on $A(N, \rho)$.

For the proof of (3.9) first consider the case $\sigma=0$ and $\rho \neq 0$. Let $z \in A(N, 0), w \in A(N, \rho)$ and $m, n \in N$, then we see by definition that $z_{k} \in A(N, 0)$ for all $k$. But for $k \geq l+2$ (where $l=$ dimension $A(N, 0)$ ) we see $z_{k}=z_{k-1} R_{n}$ and this implies $z_{l+2+k}=z_{k-1} R_{n}^{l+3}$ and so for large enough $M, z_{k}=0$ for all $k \geqq M$. This and (3.8) imply $J\left(w_{k}, m, z\right)=0$ for all $k \geqq M$. But since $w_{k}=w R_{n}^{k}$ where $w \in A(N, \rho)$ with $\rho \neq 0$, there exists $n \in N$ with $R_{n}$ nonsingular on $A(N, \rho)$ and therefore $A(N, \rho)=A(N, \rho) R_{n}^{k}$. Thus any $x \in A(N, \rho)$ is of the form $x=w R_{n}^{k}$ for some $w \in A(N, \rho)$ and therefore $J(x, m, z)=0$. But $m$ and $z$ are arbitrary in $N$ and $A(N, 0)$ respectively which implies

$$
J(A(N, \rho), A(N, 0), N)=0 .
$$

Next consider the case $0 \neq \sigma \neq \rho \neq 0$ and let $w \in A(N, \rho), z \in A(N, \sigma)$ and $m, n \in N$. Then from (3.8) we have for $k$ large enough,

$$
\begin{aligned}
J\left(w R_{n}^{k}, m, z\right) & =J\left(w_{k}, m, z\right) \\
& =J\left(w, n, z_{k}\right), \text { where } z_{k}=z_{k-1} R_{n} \\
& =J\left(w, n, z_{k-1} n\right) \\
& =-J\left(z_{k-1} n, n, w\right) \\
& =J\left(w n, n, z_{k-1}\right), \text { using }(3.3) .
\end{aligned}
$$

Therefore for $x=z_{k-1} \in A(N, \sigma)$ we have from (3.13)

$$
J\left(w, n, x\left(R_{n}-\sigma(n) I\right)\right)=J\left(w\left(R_{n}-\sigma(n) I\right), n, x\right)
$$


and by induction

$$
J\left(w, n, x\left(R_{n}-\sigma(n) I\right)^{t}\right)=J\left(w\left(R_{n}-\sigma(n) I\right)^{t}, n, x\right) .
$$

But since $x \in A(N, \sigma)$, the left side of the above equation is zero for large enough $t$ and so $J\left(w\left(R_{n}-\sigma(n) I\right)^{t}, n, x\right)=0$ for all $n \in N$. Now choose $n=u$ of Lemma 3.11, then since $\sigma(u) \neq \rho(u), R_{u}-\sigma(u) I$ is nonsigular on $A(N, \rho)$ and therefore for any integer $t>0, A(N, \rho)=$ $A(N, \rho)\left(R_{u}-\sigma(u) I\right)^{t}$. Thus any $v \in A(N, \rho)$ is of the form $v=w\left(R_{u}\right.$ $\sigma(u) I)^{t}$ and therefore $J(v, u, x)=0$ where $x$ and $u$ are defined above. Thus using the preceding notaion and (3.13) we have

$$
\begin{aligned}
0 & =J(v u, u, x), \text { since } v u \in A(N, \rho) \\
& =J\left(v u, u, z_{k-1}\right) \\
& =J\left(v, u, z_{k-1} u\right) \\
& =J\left(v R_{u}^{k}, m, z\right) .
\end{aligned}
$$

But also from Lemma 3.11, $R_{u}$ is nonsigular on $A(N, \rho)$ so that $A(N, \rho)=A(N, \rho) R_{u}^{k}$. Thus by the choise of $z, m, v$ we have

$$
J(A(N, \rho), A(N, \sigma), N)=0 \text { if } 0 \neq \sigma \neq \rho \neq 0 .
$$

Using the usual Lie algebra arguments we combine (3.12) and (3.14) to obtain

$$
A(N, \rho) A(N, \sigma) \subset A(N, \rho+\sigma) \text { if } \rho \neq \sigma .
$$

Next we prove

$$
J(A(N, \rho), A(N, \sigma), A(N, \tau))=0 \text { if } \rho \neq \sigma \neq \tau \neq \rho .
$$

For let $n \in N, x \in A(N, \rho), y \in A(N, \sigma)$ and $z \in A(N, \tau)$, then assuming first that $\rho \neq \sigma+\tau$ we have, using (3.5) and (3.12) or (3.14),

$$
\begin{aligned}
n J(x, y, z) & =3[J(n x, y, z)+J(y z, n, x)] \\
& =3 J(n x, y, z) .
\end{aligned}
$$

Therefore $J(x, y, z)\left(R_{n}-3 \rho(n) I\right)=3 J\left(x\left(R_{n}-\rho(n) I\right), y, z\right)$ and we proceed by induction to conclude

$$
J(x, y, z)\left(R_{n}-3 \rho(n) I\right)^{t}=0 \text { for large enough } t .
$$

Thus with $\rho \neq \sigma \neq \tau \neq \rho$ we conclude

$$
J(A(N, \rho), A(N, \sigma), A(N, \tau)) \subset A(N, 3 \rho) \text { if } \rho \neq \sigma+\tau .
$$

By the symmetry of the $\rho, \sigma$ and $\tau$ we also conclude

$$
\begin{gathered}
J(A(N, \rho), A(N, \sigma), A(N, \tau)) \subset A(N, 3 \sigma) \text { if } \sigma \neq \rho+\tau \text { and } \\
J(A(N, \rho), A(N, \sigma), A(N, \tau)) \subset A(N, 3 \tau) \text { if } \tau \neq \rho+\sigma .
\end{gathered}
$$


Now suppose $\rho=\sigma+\tau$. If $\sigma=\rho+\tau$, then $\tau=0$ and therefore $\sigma=\rho$, a contradiction; thus $\sigma \neq \rho+\tau$. Similarly we have $\tau \neq \rho+\sigma$ and from these we may conclude

$$
J(A(N, \rho), A(N, \sigma), A(N, \tau)) \subset A(N, 3 \sigma) \cap A(N, 3 \tau)=0 .
$$

Next suppose $\rho \neq \sigma+\tau$. If both $\sigma=\rho+\tau$ and $\tau=\rho+\sigma$ we obtain $\sigma=\tau$, a contradiction; thus $\sigma \neq \rho+\tau$ or $\tau \neq \rho+\sigma$ and using the equations involving $J(A(N, \rho), A(N, \sigma), A(N, \tau))$ we see that this expression is also zero in case $\rho \neq \sigma+\tau$. This completes the proof.

4. Some assumptions, The el-algebra identities do not appear strong enough to determine $A(N, \alpha)^{2}$ in any reasonable manner, so we assume $N$ is a precartan subalgebra such that

$$
A(N, \alpha)^{2} \subset \sum_{\beta \neq 0} A(N, \beta) \quad \text { if } \alpha \neq 0 \text {. }
$$

That is, if $x, y \in A(N, \alpha)$ and $x y=\sum_{\beta} z_{\beta}$, then no $z_{\beta}$ is in $A(N, 0)$ except $z_{\beta}=0$. By considering Lie and Malcev algebras, this is a natural assumption. Now let

$$
B=\sum_{\beta \neq 0} A(N, \beta) A(N,-\beta) \oplus \sum_{\alpha \neq 0} A(N, \alpha)
$$

where we use the usual convention that if $-\beta$ is not a weight of $N$, then $A(N,-\beta)=0$. By (3.9), $\sum_{\beta \neq 0} A(N, \beta) A(N,-\beta) \subset A(N, 0)$ and for any weight $\gamma \neq 0$ of $N$ we have

$$
\begin{aligned}
B A(N, \gamma) \subset A(N, 0) A(N, \gamma)+\sum_{\alpha \neq 0} A(N, \alpha) A(N, \gamma) \\
\subset A(N, \gamma)+A(N, \gamma) A(N, \gamma)+A(N, \gamma) A(N,-\gamma) \\
+\sum_{\alpha \neq 0, \pm \gamma} A(N, \alpha+\gamma)
\end{aligned}
$$

$\subset B$, using (4.1) .

Next from (3.15) we have for any $\beta \neq 0$,

$$
J(A(N, \beta), A(N,-\beta), A(N, 0))=0 .
$$

From this it follows that

$$
\begin{aligned}
{[A(N, \beta) A(N,-\beta)] A(N, 0) } & \subset[A(N, \beta) A(N, 0)] A(N,-\beta) \\
& +[A(N,-\beta) A(N, 0)] A(N, \beta) \\
& \subset A(N, \beta) A(N,-\beta)
\end{aligned}
$$

and so

$$
B A(N, 0) \subset \sum_{\beta \neq 0}[A(N, \beta) A(N,-\beta)] A(N, 0)+\sum_{\alpha \neq 0} A(N, \alpha) A(N, 0) \subset B .
$$

Thus $B$ is an ideal of $A$ and since $A$ is simple $B=0$ or $B=A$. This 
proves

THEOREM 4.2. If $A$ is a simple el-algebra and $N$ a precartan subalgebra such that (4.1) holds, then

1.

$$
\begin{gathered}
A=\sum_{\beta \neq 0} A(N, \beta) A(N,-B) \oplus \sum_{\alpha \neq 0} A(N, \alpha) \text { and } \\
A(N, 0)=\sum_{\beta \neq 0} A(N, \beta) A(N,-\beta) ; \text { or }
\end{gathered}
$$

2. the only weight of $N$ is 0 and $A=A(N, 0)$.

Corollary 4.3. If $A$ is an el-algebra as in Theorem 4.2, then $A(N, 0)$ is a subalgebra. Furthermore if conclusion (1) of the theorem holds, then $A(N, 0)$ is a Lie subalgebra.

Proof. If conclusion (2) holds, the result is trivial. So assume conclusion (1) holds, then $A(N, 0)=\sum_{\beta \neq 0} A(N, \beta) A(N,-\beta)$ and as before

$$
A(N, 0) A(N, 0)=\sum_{\beta \neq 0}[A(N, \beta) A(N,-\beta)] A(N, 0) \subset A(N, 0) .
$$

Next for any $\beta \neq 0$ we shall show

$$
J(A(N, \beta) A(N,-\beta), A(N, 0), A(N, 0))=0
$$

and therefore $A(N, 0)$ will be a Lie subalgebra. Let $x \in A(N, \beta), y \in$ $A(N,-\beta)$ and $s, t \in A(N, 0)$, then using $A(N, 0)^{2} \subset A(N, 0)$ and (3.15) we have

$$
\begin{aligned}
J(x y, s, t) & =J(x y, s, t)+J(s t, x, y) \\
& =J(x s, t, y)+J(t y, x, s), \text { using (3.4) } \\
& =0, \text { using (3.15) }
\end{aligned}
$$

COROLlary 4.4. If $A$ is an el-algebra as in Theorem 4.2 which satisfies conclusion (1), then the mapping $A(N, 0) \rightarrow R(A(N, 0)): m \rightarrow$ $R_{m}$ is a representation of the Lie algebra $A(N, 0)$.

Proof. From Corollary 4.3 it suffices to prove

$$
J(A(N, \beta), A(N, 0), A(N, 0))=0 \text { for any } \beta \neq 0 .
$$

Let $w \in A(N, \beta)$ and $y, z \in A(N, 0)$, then for any $n \in N$ we have

$$
\begin{aligned}
J(w n, y, z) & =J(w n, y, z)+J(y z, w, n), \text { using (3.12) } \\
& =J(w y, z, n)+J(z n, w, y) \\
& =J(z n, w, y), \text { using (3.12) }
\end{aligned}
$$


and by induction we have

$$
J\left(w R_{n}^{t}, y, z\right)=J\left(z R_{n}^{t}, w, y\right) .
$$

But for large enough $t, z R_{n}^{t}=0$ and so for any $n \in N$ and $t$ large enough $J\left(w R_{n}^{t}, y, z\right)=0$. However there exists in $n \in N$ so that $R_{n}$ and therefore $R_{n}^{t}$ is nonsingular on $A(N, \beta)$ and the usual argument proves the corollary.

Corollary 4.5. If $A$ is an el-algebra as in Theorem 4.2 which satisfies conclusion (1) for some precartan subalgebra $N$, then $A(N, 0)$ is a Cartan subalgebra provided $A(N, 0)$ is a nilpotent Lie subalgebra.

Proof. Let $M=A(N, 0)$, then from Corollary 4.4 and the hypothesis we see that $M$ is a precartan subalgebra and from $\S 2$

$$
N \subset M=\bigcap_{n \in N} A\left(R_{n}, 0\right) .
$$

Thus decompose $A=A(M, 0) \oplus \sum_{\beta \neq 0} A(M, \beta)$ relative to $R(M)$ and we must show $A(M, 0) \subset M$. Well,

$$
N \subset A(N, 0)=M \subset A(M, 0)=\bigcap_{m \in M} A\left(R_{m}, 0\right)
$$

and since $N \subset M$ we have $\cap_{m \in M} A\left(R_{m}, 0\right) \subset \cap_{n \in N} A\left(R_{n}, 0\right)=M$. Thus $A(M, 0)=M$.

As in Lie algebras we make the following

Definition. An element $u$ in an el-algebra $A$ is regular if the dimension of $A\left(R_{u}, 0\right)$ is minimal.

CoROLlary 4.6. If $A$ is an el-algebra as in Theorem 4.2 which satisfies conclusion (1) for the precartan subalgebra $N=F u$ where $u$ is regular, then $A(N, 0)$ is a Cartan subalgebra.

Proof. From Corollary 4.5 it suffices to prove $A(N, 0)$ is a nilpotent Lie subalgebra and the proof in [1, Th. 3.1] can easily be modified to prove this fact.

Next we investigate the symmetric bilinear form $(x, y)=$ trace $R_{x} R_{y}$ and impose a condition on it which is satisfied by Lie and Malcev algebras and implies assumption (4.1). Some notation: for $x \in A$ or $S, T \subset A,(x, S)$ denotes $\{(x, S): s \in S\}$ and $(S, T)$ denotes $\{(s, t): s \in S$ and $t \in T\} . \quad(x, y)$ is an invariant form if $(x y, z)$ $=(x, y z)$ for all $x, y, z \in A$. 
pose $A=A(N, 0) \oplus \sum_{\alpha \neq 0} A(N, \alpha)$, then

1. if $(x, y)$ is an invariant form, then

$$
(A(N, \alpha), A(N, \beta))=0 \text { if } \alpha+\beta \neq 0 ;
$$

2. if $(x, y)$ is a nondegenerate invariant form, then

$$
A(N, \alpha)^{2} \subset \sum_{\beta \neq 0} A(N, \beta) \quad \text { if } \alpha \neq 0 ;
$$

furthermore there exists a precartan subalgebra $N_{0}$ such that $N_{0}=F u$ where $u$ is regular and $A \neq A\left(N_{0}, 0\right)$.

Proof. (1) Suppose $(x, y)$ is an invariant form and let $x \in A(N, \alpha)$, $y \in A(N, \beta)$ and $n \in N$. Then by induction we have

$$
\left(x\left(R_{n}-\alpha(n) I\right)^{k}, y\right)=(-1)^{k}\left(x, y\left(R_{n}+\alpha(n) I\right)^{k}\right)
$$

for $k=1,2, \cdots$. But for large enough $k,\left(x\left(R_{n}-\alpha(n) I\right)^{k}, y\right)=0$, since $x \in A(N, \alpha)$ and so $\left(x, y\left(R_{n}+\alpha(n) I\right)^{k}\right)=0$. But since $\beta \neq-\alpha$, there exists $n \in N$ with $\left(R_{n}+\alpha(n) I\right)^{k}$ nonsingular on $A(N, \beta)$ and therefore $A(N, \beta)=A(N, \beta)\left(R_{n}+\alpha(n) I\right)^{k}$. This proves (1).

(2) Suppose $(x, y)$ is a nondegenerate invariant form, then there exists $n \in A$ such that $(n, n)=$ trace $R_{n}^{2} \neq 0$; otherwise by linearization, $(x, y)=0$ for all $x, y \in A$. Thus $R_{n}$ is not nilpotent and we can choose an element $u \in A$ such that $A \neq A\left(R_{u}, 0\right)$ and $u$ regular. Thus $N_{0}=F u$ is a precartan subalgebra such that $A \neq A\left(N_{0}, 0\right)$. Next let $\alpha \neq 0$ and $x, y \in A(N, \alpha)$ and $x, y \in A(N, \alpha)$ and $x y=z_{0}+\sum_{\beta \neq 0} z_{\beta}$ where $N$ is any precartan subalgebra. Now for $\gamma \neq 0$ we have

$$
\left(z_{0}, A(N, \gamma)\right)=0 \text {, using part (1) . }
$$

For $\gamma=0$ we have

$$
\begin{aligned}
\left(z_{0}, A(N, 0)=\right. & \left(x y-\sum_{\beta \neq 0} z_{\beta}, A(N, 0)\right) \\
& (x, y A(N, 0))-\sum_{\beta \neq 0}\left(z_{\beta}, A(N, 0)\right) \\
= & 0, \text { using part }(1) .
\end{aligned}
$$

Thus $\left(z_{0}, A\right)=0$ and since $(x, y)$ is nondegenerate $z_{0}=0$.

For the remainder of this paper we shall assume $(x, y)$ is a nondegenerate invariant form on $A$. With this assumption we may conclude that $A$ has a precartan subalgebra $N_{0}$ such that (4.1) actually holds, $A \neq A\left(N_{0}, 0\right)$ and $A\left(N_{0}, 0\right)$ is a Cartan subalgebra. Thus we may assume the existence of a Cartan subalgebra $N=A(N, 0) \neq A$ satisfying 4.1, and call $A$ "the usual el-algebra." We shall work with a fixed Cartan subalgebra described above and use the notation $A(\alpha)$ or $A_{\alpha}$ for $A(N, \alpha)$ and also $N$ for $A(N, 0)$. The proof of the following is similar to the proof for Lie algebras [1, Section 4.1]. 
THEOREM 4.8. Let $A$ be the usual el-algebra, then

1. $(x, y)$ is nondegenerate on $N$ and for $x, y \in N,(x, y)=\sum_{\rho} N_{\rho} \rho(x) \rho(y)$ where the sum is over all weights $\rho$ and $N_{\rho}=$ dimension $A(\rho)$.

2. $N^{2}=0$.

3. $0 \neq \rho$ is a weight if and only if $0 \neq-\rho$ is a weight; furthermore $A(\rho)$ and $A(-\rho)$ are dual relative to $(x, y)$ so that dimension $A(\rho)=$ dimension $A(-\rho)$.

4. Let $N^{*}$ be the dual space of $N$. If $g \in N^{*}$, then there exists a unique element $n_{g} \in N$ such that $g(n)=\left(n, n_{g}\right)$ for all $n \in N$. Furthermore the mapping $N^{*} \rightarrow N: g \rightarrow n_{g}$ is a bijection.

5. If $l=$ dimension $N$ over $F$, then there are l linearly independent weights of $N$ and these form a basis of $N^{*}$. Furthermore if $\rho, \sigma \in$ $N^{*}$ and $n_{\rho}, n_{\sigma}$ are determined as in (4), then the symmetric bilinear form $\langle\rho, \sigma\rangle=\left(n_{\rho}, n_{\sigma}\right)$ is nondegenerate on $N^{*}$.

6. If $\rho$ is a nonzero weight of $N$ and $x_{\rho} \in A(\rho)$ is such that

$$
x_{\rho} R_{n}=\rho(n) x_{\rho} \text { for all } n \in N
$$

and if $x_{-p}$ is any element in $A(-\rho)$, then

$$
x_{-\rho} x_{\rho}=\left(x_{\rho}, x_{-\rho}\right) n_{\rho}
$$

where $n_{\rho}$ is determined in (4).

7. $\rho\left(n_{\rho}\right) \neq 0$ if $\rho \neq 0$.

We shall prove (7) since its proof is different from the proof of the corresponding statement in [1]. First we need

LEMMA 4.9 Let $A=N \oplus \sum_{\alpha \neq 0} A(\alpha)$ be the usual el-algebra with Cartan subalgebra $N=\sum_{\alpha \neq 0} A(\alpha) A(-\alpha)$. Let $\phi$ be any weight of $N$ and $\rho$ any nonzero weight of $N$ and let $h \in \sum_{i=1}^{\infty} A(i \rho) A(-i \rho)$. Then $\phi(h)=r \rho(h)$ where $r$ is a rational number.

Proof. If $\phi=r \rho$ for some rational number, then we are finished. Otherwise $\phi \neq r \rho$ for any rational number $r$ and we consider

$$
M=\sum_{k} A(\phi+k \rho) \quad k=0, \pm 1, \pm 2, \cdots
$$

Now since there are finitely many weights we may write $h=\sum_{i=1}^{m} h_{i}$ where $h_{i} \in A(i \rho) A(-i \rho)$ and therefore $h_{i}=\sum_{j} x^{j}(i \rho) x^{j}(-i \rho)$ where $x^{j}(k \rho)$ $\in A(k \rho), k= \pm i$. Now since $h_{i} \in N, M$ is $R\left(h_{i}\right)$-invariant and so $R(h)$ -invariant. But since $\phi \neq r \rho$ for any rational number $r$, we use (3.9) to see that $M$ is also $R\left(x^{j}(i \rho)\right)$ - and $R\left(x^{j}(-i \rho)\right)$-invariant for $i=1, \cdots, m$.

Next for $z=\sum_{k} z_{k} \in M$ where $z_{k} \in A(\phi+k \rho)$ we have, using (3.15),

$$
J\left(z, x^{j}(i \rho), x^{j}(-i \rho)\right)=\sum_{k} J\left(z_{k}, x^{\jmath}(i \rho), x^{j}(-i \rho)\right)=0 \text {. }
$$

Therefore on $M$ we have 


$$
\begin{aligned}
R\left(h_{i}\right) & =\sum_{j} R\left(x^{j}(i \rho) x^{j}(-i \rho)\right) \\
& =\sum_{j}\left[R\left(x^{j}(i \rho)\right), R\left(x^{j}(-i \rho)\right)\right]
\end{aligned}
$$

so that on $M$

$$
R(h)=\sum_{i} R\left(h_{i}\right)=\sum_{i j}\left[R\left(x^{j}(i \rho)\right), R\left(x^{j}(-i \rho)\right)\right] .
$$

Therefore $\operatorname{trace}_{\mu} R(h)=0$. But since $h \in N$ we can calculate the trace of $R(h)$ on $M$ from its matrix to see that

$$
\begin{aligned}
\operatorname{trace}_{M} R(h) & =\sum_{k} N_{\phi+k \rho}(\phi+k \rho)(h) \\
& =\left(\sum_{k} N_{\phi+k \rho}\right) \phi(h)+\left(\sum_{k} k N_{\phi+k \rho}\right) \rho(h),
\end{aligned}
$$

where $N_{\phi+k \rho}=$ dimension $A(\phi+k \rho)$. The result now follows.

For the proof of 4.8.7 suppose $\rho$ is a nonzero weight of $N$ such that $\rho\left(n_{\rho}\right)=0$. Then for any $n \in N$ we have

$$
\rho(n)=\left(n, n_{\rho}\right)=\sum_{\alpha} N_{\alpha} \alpha\left(n_{\rho}\right) \alpha(n),
$$

summed over all weights $\alpha$. Now we can find an element $x_{-\rho} \in A(-\rho)$ such that $\left(x_{\rho}, x_{-\rho}\right)=1$ and therefore from 4.8.6, $n_{\rho}=x_{-\rho} x_{\rho}$ and so using Lemma 4.9 we have $\alpha\left(n_{\rho}\right)=r \rho\left(n_{\rho}\right)=0$ where $r$ is a rational number depending on $\alpha$ and $\rho$. Therefore $\rho(n)=0$ i.e. $\rho=0$, a contradiction.

Finally we use the nondegenerate invariant form $(x, y)$ to obtain more information on $A(\alpha)^{2}$.

THEOREM 4.10. Let $A$ be the usual el-algebra, then

$$
A(\alpha)^{2} \subset A(-\alpha)+A(2 \alpha) \text {. }
$$

Proof. For $\alpha=0$ we know the result. Suppose $\alpha \neq 0$; if $A(\alpha)^{2}=$ 0 , then we are finished. So assume $A(\alpha)^{2} \neq 0$, then since $(x, y)$ is nondegenerate there exists a weight $\beta$ such that

$$
\left(A(\alpha)^{2}, A(\beta)\right) \neq 0 \text {. }
$$

Case 1. If $\beta \neq \alpha$, then

$$
0 \neq\left(A(\alpha)^{2}, A(\beta)\right)=(A(\alpha), A(\alpha) A(\beta)) \subset(A(\alpha), A(\alpha+\beta)) .
$$

Thus $\alpha+\beta$ must be a weight and so by Lemma 4.7.1, $\alpha+(\alpha+\beta)=$ 0 , that is, $\beta=-2 \alpha$.

Case 2. $\beta=\alpha$.

Thus we have $\left(A(\alpha)^{2}, A(-2 \alpha)+A(\alpha)\right) \neq 0$. Now let $x, y \in A(\alpha)$ and $x y=\sum_{\sigma} z_{\sigma}$. Suppose there exists a component $z_{\gamma}$ with $\gamma \neq-\alpha$ or $2 \alpha$, then we have 


$$
\begin{aligned}
(x y, A(-\gamma)) & =(x, y A(-\gamma)) \\
& \subset(A(\alpha), A(\alpha-\gamma)), \text { since } \gamma \neq-\alpha \\
& =0, \text { since } \alpha+(\alpha-\gamma) \neq 0 .
\end{aligned}
$$

Thus

$$
\begin{aligned}
0 & =(x y, A(-\gamma)) \\
& =\sum_{\sigma}\left(z_{\sigma}, A(-\gamma)\right) \\
& =\left(z_{\gamma}, A(-\gamma)\right), \text { using Lemma 4.7.1. }
\end{aligned}
$$

Therefore by Theorem 4.8.3, $z_{\gamma}=0$ and so $x y=z_{-\alpha}+z_{2 \alpha}$.

5. Weight space subalgebras. Let $A=N \oplus \sum_{\rho \neq 0} A(\rho)$ be the usual simple el-algebra discussed in $\S 4$. Since we know - $\rho$ is a weight if and only if $\rho$ is a weight, we may eliminate superfluous weight spaces and write

$$
A=\sum_{\rho} A(-\rho) \oplus N \oplus \sum_{\rho} A(\rho)
$$

where $\rho$ is a nonzero weight. Now for a fixed nonzero weight $\rho$ we shall consider the weight space subalgebra

$$
B(\rho)=\sum_{k=1}^{\infty} A(-k \rho) \oplus \sum_{k=1}^{\infty} A(-k \rho) A(k \rho) \oplus \sum_{k=1}^{\infty} A(k \rho)
$$

and show it actually equals

$$
A(-\rho) \oplus n_{\rho} F \oplus A(\rho)
$$

where $n_{\rho}$ determined in Theorem 4.8.4. In the next section we shall show $B(\rho)$ is the split 3-dimensional simple Lie algebra or the simple 7-dimensional Malcev algebra obtained from the split Cayley-Dickson algebra.

Proposition 5.1. Let $B(\rho)$ be a weight space subalgebra, then $\sum_{k=1}^{\infty} A\left(-k_{k} \rho\right) A(k \rho)=n_{\rho} F$.

Proof. Let $B=\sum_{k} A\left(-k_{e} \rho\right) A(k \rho)$, then it suffices to show dimension $B=1$; for $0 \neq n_{\rho} \in B$ and we would have $B=n_{\rho} F$. To show this let $B^{*}$ denote the dual space of $B$ and prove dimencion $B^{*}=1$. Since dimension $B=$ dimension $B^{*}$ we have the results, Let $x \in A$ be such that for any $a \in B(\rho), a x \in B(\rho)$ and set

$$
\bar{R}_{x}: B(\rho) \rightarrow B(\rho): a \rightarrow a x .
$$

Let $x, y \in B(\rho)$ and set 


$$
f(x, y)=\operatorname{trace} \bar{R}_{x} \bar{R}_{y}\left(=\operatorname{trace}_{B(\rho)} R_{x} R_{y}\right) .
$$

$f(x, y)$ is a bilinear form on $B(\rho)$ and the fact that dimension $B^{*}=1$ is a consequence of the following lemma.

Lemma 5.2. 1. $f(x, y)$ is nondegenerate on $B$ and 2. dimension $B^{*}=1$.

Proof. Since there are finitely many weights we can apply Theorem 4.8 to show that for any $h, k \in B, f(h, k)=\operatorname{trace} \bar{R}(h) \bar{R}(k)=$

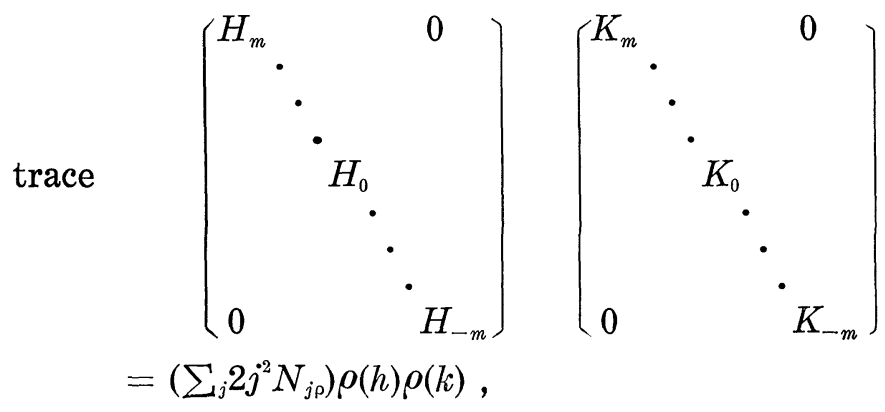

where

$$
H_{j}=\left(\begin{array}{ccc}
j \rho(h) & & 0 \\
& \cdot & \\
* & & j \rho(h)
\end{array}\right) \text { and } K_{j}=\left(\begin{array}{lll}
j \rho(k) & & 0 \\
& \cdot & \\
* & & j \rho(k)
\end{array}\right)
$$

Now suppose there exists $b \in B$ such that for all $k \in B$

$$
0=f(b, k)=\left(\sum_{j} 2 j^{2} N_{j \rho}\right) \rho(b) \rho(k) .
$$

Set $k=b$ to conclude $\rho(b)=0$. Now since $b \in B=\sum_{j} A(j \rho) A(-j \rho)$ we have form Lemma 4.9 that for any weight $\phi$ of $N$ in $A, \phi(b)=$ $r \rho(b)=0$. Now for any $x \in N$ we can apply Theorem 4.8.1 to conclude $(x, b)=0$ and therefore $b=0$. Thus $f(x, y)$ is nondegenerate on $B$.

Next let $g \in B^{*}$, then since $f(x, y)$ is nondegenerate on $B$, there exists a unique $b \in B$ so that for all

$$
h \in B g(h)=f(h, b)=\left(\sum_{j} 2 j^{2} N_{j \rho}\right) \rho(b) \rho(h)=c \rho(h)
$$

where $c \in F$. Thus $B^{*}=\rho F$ is of dimension one.

Corollary 5.3. $B(\rho)=\sum_{k} A(-k \rho) \oplus n_{\rho} F \oplus \sum_{k} A(k \rho)$.

Put $R\left(n_{\rho}\right)$ into its Jordan canonical form on $A(-\rho)$. Thus we can write 


$$
A(-\rho)=U(1,-\rho) \oplus \cdots \oplus U(k,-\rho)
$$

where each $U(j,-\rho)$ has a basis $\left\{z_{j_{1}}, \cdots, z_{j_{m_{j}}}\right\}$ such that, if $\rho \equiv \rho\left(n_{\rho}\right)$,

$$
\begin{aligned}
& z_{j 1} R\left(n_{\rho}\right)=-\rho z_{j 1} \quad \text { and } \\
& z_{j i} R\left(n_{\rho}\right)=-\rho z_{j i}+z_{j i-1}
\end{aligned}
$$

$i=2,3, \cdots, m_{\jmath}$. We shall consider just one of the $R\left(n_{\rho}\right)$-invariant spaces $U(j,-\rho)$ and denote it by $U \equiv\left\{z_{1}, \cdots, z_{m}\right\}$. We use symmetry to argue similar results for the other spaces and also for the weight $\rho$.

Lemma 5.5. Let $x \in A(\rho)$ be such that $x R\left(n_{\rho}\right)=\rho x$, then $z_{i} x=$ $\lambda_{i} n_{\rho}$ and $\lambda_{i}=0$ for $i=1, \cdots, m-1$.

Proof. From Proposition $5.1 z_{i} x=\lambda_{i} n_{\rho}$ and from (3.15) we have for $i=2, \cdots, m$ that

$$
\begin{aligned}
0=J\left(z_{i}, x, n_{\rho}\right) & =z_{i} x \cdot n_{\rho}+x n_{\rho} \cdot z_{i}+n_{\rho} z_{i} \cdot x=\rho x z_{i}-z_{i} n_{\rho} \cdot x \\
& =\rho x z_{i}-\left(-\rho z_{i}+z_{i-1}\right) x=z_{i-1} x
\end{aligned}
$$

also using Theorem 4.8.2. Thus $\lambda_{i}=0, i=1, \cdots, m-1$.

Proposition 5.6. $J\left(z_{i}, z_{\jmath}, n_{\mathrm{p}}\right)=0$ for $i, j=1, \cdots, m$.

Proof. Using the skew-symmetry of $J(a, b, c)$, it suffices to show $J\left(z_{i}, z_{j}, n_{\rho}\right)=0$ for $i \geqq j$ and $j=m, m-1, \cdots, 1$. We use induction on $j$. First for $j=m, J\left(z_{m}, z_{m}, n_{\rho}\right)=0$. Next for $j=m-1$ we have

$$
\begin{aligned}
J\left(z_{m}, z_{m-1}, n_{\rho}\right) & =J\left(z_{m}, z_{m}\left(R\left(n_{\rho}\right)+\rho I\right), n_{\rho}\right) \\
& =J\left(z_{m}, z_{m} n_{\rho}, n_{\rho}\right) \\
& =0, \text { using }(3.1) .
\end{aligned}
$$

Now assume that for all $j=m, m-1, \cdots, m-q$ we have shown $J\left(z_{j}, z_{j}, n_{\rho}\right)=\cdots=J\left(z_{m}, z_{j}, n_{\rho}\right)=0$, then we must show similar results for $j-1=m-(q+1)$. We always have $J\left(z_{j-1}, z_{j-1}, n_{p}\right)=0$ and next

$$
\begin{aligned}
J\left(z_{j}, z_{j-1}, n_{\rho}\right) & =J\left(z_{j}, z_{j}\left(R\left(n_{\rho}\right)+\rho I\right), n_{\rho}\right) \\
& =J\left(z_{j}, z_{j} n_{\rho}, n_{\rho}\right)=0 .
\end{aligned}
$$

So we now assume

$$
J\left(z_{j-1}, z_{j-1}, n_{\rho}\right)=\cdots=J\left(z_{j-1+k}, z_{i-1}, n_{\rho}\right)=0
$$

for $j-1<j-1+k<m$; we then have 


$$
\begin{aligned}
J\left(z_{j-1}, z_{j+k}, n_{\rho}\right) & =J\left(z_{j}\left(R\left(n_{\rho}\right)+\rho I\right), z_{j+k}, n_{\rho}\right) \\
& =J\left(z_{j} n_{\rho}, z_{j+k}, n_{\rho}\right)+\rho J\left(z_{j}, z_{j+k}, n_{\rho}\right) \\
& =J\left(z_{j} n_{\rho}, z_{j+k}, n_{\rho}\right), \text { using the first induction } \\
& =-J\left(z_{j} n_{\rho}, n_{\rho}, z_{j+k}\right) \\
& =J\left(z_{j+k} n_{\rho}, n_{\rho}, z_{j}\right), \text { using (3.3) } \\
& =-\rho J\left(z_{j+k}, n_{\rho}, z_{j}\right)+J\left(z_{j-1+k}, n_{\rho}, z_{j}\right) \\
& =0 .
\end{aligned}
$$

Proposition 5.7. For all $n \in N, J\left(z_{i}, z_{j}, n\right)=0$ and $U^{2} \subset A(-2 \rho)$.

Proof. It suffices to show that for any weight $\sigma \neq 0$ we have $J\left(z_{i}, z_{j}, n_{\sigma}\right)=0$ where $n_{\sigma}$ is determined in Theorem 4.8.4; for we see that the elements $n_{\sigma}$ span $N$ as a vector space. Now if $\sigma=\lambda \rho$ where $\lambda \in F$, it is easy to see that $n_{\sigma}=\lambda n_{\rho}$ and so from Proposition 5.6 $J\left(z_{i}, z_{j}, n_{\sigma}\right)=0$. Next assume $\sigma$ is not a scalar multiple of $\rho$ and choose elements $x_{\sigma}$ and $x_{-\sigma}$ as in Theorem 4.8 .6 so that $x_{-\sigma} x_{\sigma}=n_{\sigma}$. Now noting that $z_{i} z_{j} \in A(-\rho)^{2} \subset A(\rho) \oplus A(-2 \rho)$ we have, using 3.15,

$$
\begin{aligned}
J\left(z_{i} z_{j}, x_{-\sigma}, x_{\sigma}\right) & \in J(A(\rho), A(-\sigma), A(\sigma)) \\
& +J(A(-2 \rho), A(-\sigma), A(\sigma))=0 .
\end{aligned}
$$

Thus we have

$$
\begin{aligned}
J\left(n_{\sigma}, z_{i}, z_{j}\right) & =J\left(x_{-\sigma} x_{\sigma}, z_{i}, z_{j}\right)+J\left(z_{i} z_{j}, x_{-\sigma}, x_{\sigma}\right) \\
& =J\left(x_{-\sigma} z_{i}, z_{j}, x_{\sigma}\right)+J\left(z_{j} x_{\sigma}, x_{-\sigma}, z_{i}\right) \\
& =0 \text {, using (3.15). }
\end{aligned}
$$

Next let $u, v \in U$, then by linearity $J\left(u, v, n_{\rho}\right)=0$ and since $U$ is $R\left(n_{\rho}\right)$-invariant subspace, the usual argument shows $(u v)\left(R\left(n_{\rho}\right)+2 \rho I\right)^{k}$ $=0$ for $k$ large enough. But we know $u v=x+y$ where $x \in A(\rho), y \in$ $A(-2 \rho)$ and therefore for $k$ large enough,

$$
0=(u v-y)\left(R\left(n_{\rho}\right)+2 \rho I\right)^{k}=x\left(R\left(n_{\rho}\right)+2 \rho I\right)^{k} .
$$

If $x \neq 0$, this implies there exists $0 \neq z \in A(\rho)$ so that $z\left(R\left(n_{\mathrm{\rho}}\right)+\right.$ $2 \rho I)=0$ and therefore $-3 \rho z=z\left(R\left(n_{\rho}\right)-\rho I\right)$. Iterating this formula and using $\rho \equiv \rho\left(n_{\rho}\right) \neq 0$ we obtain $z=0$, a contradiction. Thus $x=0$ which proves the second part.

Proposition 5.8. Let $x \in A(\rho)$ be such that $x R\left(n_{\rho}\right)=\rho x$, then $J\left(z_{i}, z_{j}, x\right)=0$ for $i, j=1, \cdots, m$.

Proof. It suffices to show $J\left(z_{i}, z_{j}, x\right)=0$ for all $i \geqq j$ and 
$j=m, \cdots, 1$. The case $j=m$ is trivial and for $j=m-1$ we have

$$
\begin{aligned}
J\left(z_{m}, z_{m-1}, x\right) & =J\left(z_{m}, z_{m}\left(R\left(n_{\rho}\right)+\rho I\right), x\right) \\
& =J\left(z_{m}, z_{m} n_{\rho}, x\right) \\
& =J\left(n_{\rho} z_{m}, z_{m}, x\right) \\
& =-J\left(x z_{m}, z_{m}, n_{\rho}\right) \text {, using (3.3) } \\
& =0 \text {, using Lemma 5.5. }
\end{aligned}
$$

Assume we have shown for $j=m, m-1, \cdots, m-k$

$$
J\left(z_{i}, z_{j}, x\right)=0 \text { for } i=j, j+1, \cdots, m .
$$

We shall show for $j=m-(k+1)$ that

$$
J\left(z_{i}, z_{j}, x\right)=0 \text { for } i=j, j+1, \cdots, m .
$$

As above, the cases $i=j$ and $i=j+1$ are clear. So assume

$$
J\left(z_{i}, z_{j}, x\right)=0 \text { for } i=j, j+1, \cdots, j+t-1
$$

where $j<j+t-1<m$. Then for $i+1=j+t$ we have

$$
\begin{aligned}
J\left(z_{j+t}, z_{j}, x\right) & =J\left(z_{j+t}, z_{j+1}\left(R\left(n_{\rho}\right)+\rho I\right), x\right) \\
& =J\left(z_{j+t}, z_{j+1} n_{\rho}, x\right)+\rho J\left(z_{j+t}, z_{j+1}, x\right) \\
& =J\left(z_{j+t}, z_{j+1} n_{\rho}, x\right),
\end{aligned}
$$

using the first induction hypothesis

$$
=-\left[J\left(z_{j+1} n_{\rho}, z_{j+t}, x\right)+J\left(z_{j+t} x, z_{j+1}, n_{\rho}\right)\right] \text {, }
$$

using Lemma 5.5

$=-\left[J\left(z_{j+1} z_{j+t}, x, n_{\rho}\right)+J\left(x n_{\rho}, z_{j+1}, z_{j+t}\right)\right]$

$=-J\left(x n_{\rho}, z_{j+1}, z_{j+t}\right)$, using Proposition 5.7

$=\rho J\left(z_{j+t}, z_{j+1}, x\right)$

$=0$, using induction hypothesis.

This proves the result.

To use the preceding results we need the following remark: Let $V$ be an $R\left(n_{\rho}\right)$-invariant subspace of the weight space $A(\sigma)$, then $\operatorname{trace}_{V} R\left(n_{\mathrm{p}}\right)=\sigma\left(n_{\mathrm{p}}\right) \mu$ where $\mu$ is the dimension of $V$.

Proposition 5.9. Let $x \in A(\rho)$ be such that $x R\left(n_{\rho}\right)=\rho x$ and suppose $x U \neq 0$, where we use the notation $U \equiv\left\{z_{1}, \cdots, z_{m}\right\}$ preceding Lemma 5.5. Then the dimension of $U$ is one.

Corollary 5.10. If $x$ is given as above and $x U(j,-\rho) \neq 0$, then the dimension of $U(j,-\rho)$ is one.

Proof. Let $U_{1} \equiv U=\left\{z_{1}, \cdots, z_{m}\right\}$ denote the set of elements of 
$A(-\rho)$ we have been considering. Set $U_{2}=U U \subset A(-2 \rho)$, using Proposition 5.7; and set $U_{k}=U_{k-1} U \subset A(-k \rho)$. Then since there are finitely many weights, there exists an integer $M$ so that $U_{M} \neq 0$ but $U_{M+1}=0$. Let $P=x F \oplus n_{\rho} F \oplus \sum_{k=1}^{M} U_{k}$, then we shall show: (1) $P$ is $R_{x^{-}}, R\left(n_{\rho}\right)$ - and $R\left(z_{m}\right)$-invariant; (2) $J\left(z, x, z_{m}\right)=0$ for all $z \in P$. Then from Lemma 5.5 and the hypothesis we see $x z_{m}=\lambda n_{\rho}$ where $\lambda \neq 0$ and using the standard Lie algebra trace argument we shall show that the dimension of $U$ is one.

Suppose (1) and (2) have been proved and consider the trace argument. From (2) we see

$$
0=J\left(z, x, z_{m}\right)=z\left(\left[R(x), R\left(z_{m}\right)\right]-R\left(x z_{m}\right)\right) .
$$

Thus as linear transformations on $P$ we have

$$
\begin{aligned}
R\left(n_{\rho}\right) & =1 / \lambda R\left(x z_{m}\right), \text { since } \lambda \neq 0 \\
& =1 / \lambda\left[R(x), R\left(z_{m}\right)\right]
\end{aligned}
$$

and so $\operatorname{trace}_{P} R\left(n_{\rho}\right)=0$. But from the matrix of $R\left(n_{\rho}\right)$ on $P$ we see trace $_{P} R\left(n_{\rho}\right)=\left(\rho+0-\mu_{1} \rho-2 \mu_{2} \rho-\cdots-M \mu_{M} \rho\right)$ where $\mu_{i}$ is the dimension of $U_{i}$. Thus since $\rho \equiv \rho\left(n_{\rho}\right) \neq 0, \mu_{1}=1, \mu_{2}=\cdots=\mu_{M}=0$.

We now prove (2). If $z=x$ or $z=n_{\rho}$, the result follows. If $z \in U_{1}$, then use Proposition 5.8. Finally if $z \in U_{i}$ for $i>1$, then $z \in A(-i \rho)$ and so $J\left(z, x, z_{m}\right) \in J(A(-i \rho), A(\rho), A(-\rho))=0$. Now for an arbitrary element $z \in P$ use the above and linearity.

Next we prove (1). Since $z_{m} \in U_{1}$, each $U_{i}=U_{i-1} U_{1}$ is such that $U_{i} z_{m} \subset U_{i} U_{1}=U_{i+1}$. Furthermore since $x z_{m}=\lambda n_{\rho}$ and $n_{\rho} z_{m} \in U_{1}$ we see $P$ is $R\left(z_{m}\right)$-invariant. Next we shall show $P$ is $R\left(n_{\rho}\right)$-invariant. First $x R\left(n_{\rho}\right), n_{\rho} R\left(n_{\rho}\right)$ and $U_{1} R\left(n_{\rho}\right)$ are all contained in $P$. We have $U_{1} R\left(n_{\rho}\right) \subset U_{1}$; next assume $U_{i} R\left(n_{\rho}\right) \subset U_{i}$. Then for $U_{i+1} R\left(n_{\rho}\right)$ we note that any $a \in U_{i+1}$ is of the form $a=\sum_{j} a_{i j} z_{j}$ where $a_{i j} \in U_{i}, z_{j} \in U_{1}$. Since $U_{i} \subset A(-i \rho)$ we have

$$
\begin{aligned}
0 & =J\left(a_{i j}, z_{j}, n_{\rho}\right), \text { using }(3.15) \text { or Prosposition } 5.6 \\
& =\left(a_{i j} z_{j}\right) n_{\rho}+\left(z_{j} n_{\rho}\right) a_{i j}+\left(n_{\rho} a_{i j}\right) z_{j} .
\end{aligned}
$$

Therefore

$$
\begin{aligned}
a R\left(n_{\rho}\right) & =\sum_{j}\left(a_{i j} z_{j}\right) n_{\rho} \\
& =\sum_{j} a_{i j}\left(z_{j} n_{\rho}\right)+z_{j}\left(n_{\rho} a_{i j}\right) .
\end{aligned}
$$

Now $a_{i j}\left(z_{j} n_{\rho}\right) \in U_{i} U_{1} \subset U_{i+1}$ and by induction hypothesis $n_{\rho} a_{i j} \in U_{i}$ and therefore $z_{j}\left(n_{\rho} a_{i j}\right) \in U_{i+1}$. Thus $U_{i+1} R\left(n_{\rho}\right) \subset U_{i+1}$ and $P$ is $R\left(n_{\rho}\right)$ invariant.

Finally we shall show $P$ is $R(x)$-invariant. The elements $x R(x)$, $n_{\rho} R(x)$ and $z_{i} R(x)=\lambda_{i} n_{\rho}$ are all in $P$. Thus it suffices to show $U_{i} R(x) \subset$ 
$P$ for $i>1$. Now from Proposition 5.8 we have

$$
\begin{aligned}
0 & =J\left(z_{i}, z_{j}, x\right) \\
& =\left(z_{i} z_{j}\right) x+\left(z_{j} x\right) z_{i}+\left(x z_{i}\right) z_{j} \\
& =\left(z_{i} z_{j}\right) x+\lambda_{j} n_{\rho} z_{i}-\lambda_{i} n_{\rho} z_{j} .
\end{aligned}
$$

Thus $\left(z_{i} z_{j}\right) R(x)=\lambda_{i} n_{\rho} z_{j}-\lambda_{j} n_{\rho} z_{i} \in U_{1}$ which yields $U_{2} R(x) \subset U_{1}$. Now assume $U_{i} R(x) \subset U_{i-1}$, then since $U_{i+1}=U_{i} U_{1}$ and $U_{i} \subset A(-i \rho)$ we have for any $a=\sum a_{i j} z_{j} \in U_{i+1}$ that

$$
\begin{aligned}
0 & =J\left(a_{i j}, z_{j}, x\right) \\
& =\left(a_{i j} z_{j}\right) x+\left(z_{j} x\right) a_{i j}+\left(x a_{i j}\right) z_{j} \\
& =\left(a_{i j} z_{j}\right) x+\lambda_{j} n_{\rho} a_{i j}+b_{i j} z_{j}
\end{aligned}
$$

where by the induction hypothesis $b_{i j}=x a_{i j} \in U_{i-1}$. Thus we conclude $U_{i+1} R(x) \subset U_{i}$.

Proposition 5.11. Let $A(-\rho)=U(1,-\rho) \oplus \cdots \oplus U(k,-\rho)$ be the decomposition of $A(-\rho)$ when $R\left(n_{\rho}\right)$ is put into Jordan canonical form. Then the dimension of all the $U(i,-\rho)$ is one.

Proof. Suppose there exists $U(j,-\rho) \equiv\left\{y_{1}, \cdots, y_{m}\right\}$ of dimension $m>1$. Then putting $R\left(n_{\rho}\right)$ into its Jordon canonical form on $A(\rho)$, that is, writing $A(\rho)=U(1, \rho) \oplus \cdots \oplus U(q, \rho)$ we see $y_{1} U(k, \rho)=0$ for every $k=1, \cdots, q$. For otherwise by an argument similar to that in Proposition 5.9 we have the situation $y_{1} R\left(n_{\rho}\right)=-\rho y_{1}$ and $y_{1} U(k, \rho) \neq 0$ for some $k$ and therefore the dimension of $U(k, \rho)$ is one. But this means $U(k, \rho)=x F$ where $x R\left(n_{\rho}\right)=\rho x$ and $0 \neq x y_{1} \in x U(j,-\rho)$. So again by Proposition 5.9, the dimension of $U(j,-\rho)$ is one, a contradiction. Thus we have $y_{1} U(k, \rho)=0$ for all $k$ and therefore $y_{1} A(\rho)=$ 0 . But using the nondegenerate invariant form $(u, v)$ this yields for every $z \in A(\rho),-\rho\left(z, y_{1}\right)=\left(z, y_{1} n_{\rho}\right)=\left(z y_{1}, n_{\rho}\right)=0$. Thus $\left(A(\rho), y_{1}\right)=0$ and since $y_{1} \in A(-\rho)$ we have $y_{1}=0$, a contradiction.

Proposition 5.11 means $R\left(n_{\rho}\right)$ can be diagonalized on $A(-\rho)$ and by a symmetrical argument $R\left(n_{\rho}\right)$ can be diagonalized on $A(\rho)$. Similarly $R\left(n_{k \rho}\right)$ can be diagonalized on $A( \pm k \rho)$. But since $n_{k \rho}=k n_{\rho}$ we see $R\left(n_{\rho}\right)$ can be diagonalized on $A( \pm k \rho)$ and therefore on the weight space subalgebra $B(\rho)$. We use these facts in the remainder of this section to show that $B(\rho)=A(-\rho) \oplus n_{\rho} F \oplus A(\rho)$.

Let $v$ be a fixed element in $A(\rho)$ where $\rho$ is any nonzero weight and let

$$
E(v)=\{x \in A(\rho): x v \in A(2 \rho)\} .
$$

Since $v^{2}=0 \in A(2 \rho), v \in E(v)$. We shall now show 
LEMMA 5.12.

$$
E(v)=v F
$$

Proof. Clearly $E \equiv E(v)$ is a subspace. Now let $y \in A(-\rho)$ be such that $n_{\rho}=y v$ and let

$$
Q=y F \oplus n_{\rho} F \oplus E \oplus E R(v) \oplus \cdots \oplus E R(v)^{t}
$$

where $t$ is such that $E R(v)^{t} \neq 0$ but $E R(v)^{t+1}=0$. We shall show (1) $Q$ is $R\left(n_{\rho}\right)$-, $R(y)$ - and $R(v)$-invariant; (2) $J(q, v, y)=0$ for all $q \in Q$. Then by the usual trace argument we shall show the dimension of $E$ is one which proves the result.

(1). $Q$ is $R\left(n_{\rho}\right)$-invariant. First $y R\left(n_{\rho}\right), n_{\rho} R\left(n_{\rho}\right)$ and $E R\left(n_{\rho}\right)$ are all in $Q$. Now if $x \in E$ then $z=x R^{j}(v) \in E R^{j}(v) \subset A((j+1) \rho)$. Since $R\left(n_{\rho}\right)$ acts diagonally in $A((j+1) \rho)$ we have $z R\left(n_{\rho}\right)=(j+1) \rho z \in E R^{j}(v)$. Next $Q$ is $R(v)$-invariant by the choice of $y$ and since $v \in E$. Finally $Q$ is $R(y)$-invariant. We know $y R(y), n_{\rho} R(y)$ and $E R(y)$ are all in $Q$, noting $E R(y) \subset n_{\rho} F$. So let $x \in E$, then we shall show $(x R(v)) R(y) \in E$ and therefore $(E R(v)) R(y) \subset E$. We have

$$
\begin{aligned}
-\rho J(y, x, v) & =J\left(y n_{\rho}, x, v\right) \\
& =J\left(y n_{\rho}, x, v\right)+J\left(x v, y, n_{\rho}\right), \text { using } x v \in A(2 \rho) \text { and } \\
& =J\left(y x, v, n_{\rho}\right)+J\left(v n_{\rho}, y, x\right) \\
& =\rho J(v, y, x), \text { since } y x \in n_{\rho} F \\
& =\rho J(y, x, v) .
\end{aligned}
$$

Therefore $J(y, x, v)=0$ and from this, $(x v) y=(x y) v+x(v y) \in v F+$ $x F \subset E$. Now assume $\left(E R(v)^{\jmath}\right) R(y) \subset E R(v)^{j-1}$, then for $w=z R(v) \epsilon$ $E R(v)^{j+1}$ where $z \in E R(v)^{j} \subset A((j+1) \rho)$ we have $0=J(z, v, y)=$ $(z v) y+(v y) z+(y z) v$. From this we obtain

$$
w R(y)=(z v) y=-(v y) z-(y z) v=n_{\rho} z+z^{\prime} v \in E R(v)^{j}
$$

where $z^{\prime}=y z \in E R(v)^{j-1}$ using the induction hypothesis. Thus by the choice of $w$ we have $\left(E R(v)^{j+1}\right) R(y) \subset E R(v)^{j}$.

Next we prove (2). First if $q=y$ or $q=n_{\rho}$, then $J(q, v, y)=0$. Now for $q \in E$ we see from the above proof that $J(q, v, y)=0$ (by the change of notation of $x$ to $q)$. Now for $q \in E R(v)^{j} \subset A((j+1) \rho)$ we have by (3.15), $J(q, v, y)=0$. Thus by linearity we have for all $q \in Q$, $J(q, v, y)=0$.

Next we apply the standard trace argument. On $Q$ we have from (2) that

$$
q([R(v), R(y)]-R(v y))=0
$$

so that on $Q$ we have $R\left(n_{\rho}\right)=[R(y), R(v)]$ and therefore $\operatorname{trace}_{Q} R\left(n_{\rho}\right)=0$. 
But from the matrix of $R\left(n_{\rho}\right)$ on $Q$, remembering $R\left(n_{\rho}\right)$ acts diagonally on $B(\rho)$, we see $\operatorname{trace}_{Q} R\left(n_{\rho}\right)=-\rho+0+\mu_{0} \rho+2 \mu_{1} \rho+\cdots+(t+1) \mu_{t} \rho$ where $\mu_{j}$ is the dimension of $E R(v)^{j}$. Thus since $\rho \neq 0, \mu_{0}=1$ and $\mu_{1}=\cdots=\mu_{t}=0$.

THEOREM 5.13. Let $\rho$ be a nonzero weight, then the only integral multiples of $\rho$ which are weights are $0, \pm \rho$. Thus the weight space subalgebra $B(\rho)=A(-\rho) \oplus n_{\rho} F \oplus A(\rho)$ and therefore $A(\rho)^{2} \subset A(-\rho)$.

Proof. Let $x \in A(\rho)$ and $y \in A(-\rho)$ be such that $x y=n_{\rho}$ and let

$$
R=x F \oplus n_{\rho} F \oplus y F \oplus \sum_{k=2}^{\infty} A(-k \rho) .
$$

Then $R$ is $R\left(n_{\rho}\right)$ - and $R(y)$-invariant. Next we shall show $R$ is $R(x)$ -invariant. Clearly the elements $x R(x), n_{\rho} R(x), y R(x)$ and $A(-k \rho) R(x)$ for $k \geqq 3$ are all in $R$. So we must show $A(-2 \rho) R(x) \subset R$. For any $z \in A(-2 \rho)$ we have

$$
0=J(z, x, y)=(z x) y+(x y) z+(y z) x=(z x) y+n_{\rho} z+(y z) x
$$

and from this $(z x) y \in A(-2 \rho)+A(-3 \rho) A(\rho) \subset A(-2 \rho)$. Thus the element $z x \in A(-2 \rho) A(\rho) \subset A(-\rho)$ is contained in the set $E(y)$ discussed in Lemma 5.12 and $E(y)$ equals $y F$. Thus $A(-2 \rho) R(x) \subset y F \subset R$ so that $R$ is $R(x)$-invariant.

Now for any $q \in R, J(q, x, y)=0$ and therefore $R\left(n_{\rho}\right)=R(x y)=$ $[R(x), R(y)]$ on $R$ so that $\operatorname{trace}_{R} R\left(n_{\rho}\right)=0$. But from the matrix of $R\left(n_{\rho}\right)$ on $R$ we see

$$
\operatorname{trace}_{R} R\left(n_{\rho}\right)=\rho+0-\rho+2 \mu_{2} \rho+3 \mu_{3} \rho+\cdots
$$

where $\mu_{k}$ is the dimension of $A(-k \rho), k \geqq 2$. Thus since $\rho \neq 0$ we must have $\mu_{k}=0$ for $k \geqq 2$ which shows $A(-k \rho)=0$ and by Theorem 4.8.3, $A(k \rho)=0$ for $k \geqq 2$. The fact that $A(\rho)^{2} \subset A(-\rho)$ now follows from Theorem 4.10.

6. More on weight space subalgebras. In this section we continue the discussion of weight space subalgebras and prove the theorem mentioned in the introduction. Let $B(\rho)=A(\rho) \oplus n F \oplus A(-\rho)$ be a weight space subalgebra and $\left\{x_{1}, \cdots, x_{m}\right\}$ a basis for $A(\rho)$. Since $A(-\rho)$ is dual to $A(\rho)$ relative to $(x, y)=\operatorname{trace} R_{x} R_{y}$ choose $\left\{y_{1}, \cdots, y_{m}\right\}$ to be a dual basis for $A(-\rho)$ so that we have $\left(x_{i}, y_{j}\right)=\delta_{i j}$. From this we also have

$$
y_{j} x_{i}=\delta_{i j} n_{\rho}
$$


For by Proposition 5.1 we have $y_{j} x_{i}=\lambda n_{\rho}$ and therefore $\lambda \rho=\lambda\left(n_{\rho}, n_{\rho}\right)$ $=\left(\lambda n_{\rho}, n_{\rho}\right)=\left(y_{j} x_{i}, n_{\rho}\right)=\left(y_{j}, x_{i} n_{\rho}\right)=\rho\left(y_{j}, x_{i}\right)=\rho \delta_{i j}$.

Next let $x \in A$ be such that for all $a \in B(\rho), a x \in B(\rho)$ and set $\bar{R}_{x}: a \rightarrow a x$ (see Proposition 5.1) and let $B(x, y)=(1 / 2 m \rho)$ trace $\bar{R}_{x} \bar{R}_{y}$, then we have

TheOREM 6.2. $B(\rho)$ is a simple subalgebra of $A$ and $B(x, y)$ is a nondegenerate invariant form on $B(\rho)$.

Proof. Let $C \neq 0$ be an ideal of $B(\rho)$ containing an element $c=\Sigma c_{i} x_{i}+c_{0} n_{\rho}+\Sigma c_{i}^{\prime} y_{i}$. First assume $c_{0} \neq 0$, then from the multiplicative relations in $B(\rho)$ we have $\rho^{2} c-\left(c n_{\rho}\right) n_{\rho}=\rho^{2} c_{0} n_{\rho} \in C$ and therefore $n_{\rho} \in C$ which implies $C=B$, using $A( \pm \rho) n_{\rho} \subset A( \pm \rho)$. Next we shall show that $C$ always contains an element with a nonzero coefficient for $n_{\rho}$. Suppose $c=\Sigma c_{i} x_{i}+\Sigma c_{i}^{\prime} y_{i} \in C$ and assume some $c_{k} \neq 0$, then $c y_{k}=\Sigma c_{i} x_{i} y_{k}+\Sigma c_{i}^{\prime} y_{i} y_{k}=c_{k} x_{k} y_{k}+c_{\rho}=-c_{k} n_{\rho}+c_{\rho}$ is in $C$ where $c_{\rho} \in A(\rho)$ and $c_{k} \neq 0$. Similarly if some $c^{\prime}{ }_{k} \neq 0$. Thus by the first of the proof $B(\rho)$ is simple.

To show $B(x, y)$ is a nondegenerate invariant from on $B(\rho)$, it suffices by Lemma 5.2 to show $B(x, y)$ is an invariant form; for in this case $\{x \in B(\rho): B(x, B(\rho))=0\}$ is an ideal of $B(\rho)$ which must be zero. From identities (3.4) we have

$$
\text { trace } \bar{R}(x y) \bar{R}(z)-\operatorname{trace} \bar{R}(x) \bar{R}(y z)=\operatorname{trace} \bar{R}(x y \cdot z+x \cdot y z),
$$

since $B(\rho)$ is a subalgebra and therefore satisfies the same indentities as $A$. Thus it suffices to show trace $\bar{R}(z)=0$ for all $z \in B(\rho)$. We have trace $\bar{R}\left(n_{\rho}\right)=0$ since dimension $A(\rho)=$ dimension $A(-\rho)$ and by the action of $\bar{R}\left(n_{\rho}\right)$ on $B(\rho)$. Similarly by the multiplicative relations of any basis element $x_{\rho}$ of $A(\rho)$ in $B(\rho)$ we see that $R\left(x_{\rho}\right)$ has a matrix of trace zero; the same holds for $R\left(x_{-\rho}\right)$. Thus by linearity of the trace function we have the results.

Next we investigate the identities for $B(\rho)$ more closely.

$$
(x y) z= \begin{cases}B(x y, z) n_{\rho} & \text { if } x, y, z \in A(\rho) \\ -B(x y, z) n_{\rho} & \text { if } x, y, z \in A(-\rho)\end{cases}
$$

For, by the multiplicative relations in $B(\rho)$, there exists $\lambda \in F$ with $(x y) z=$ $\lambda n_{\rho}$. Since $B\left(n_{\rho}, n_{\rho}\right)=\rho \neq 0$ we have $\lambda \rho=B\left(\lambda n_{\rho}, n_{\rho}\right)=B\left(x y, z, n_{\rho}\right)$ $=B\left(x y, z n_{\rho}\right)$ and this implies the result. Using (6.3) we have

$$
J(x, y, z)= \begin{cases}3 B(x y, z) n_{\rho} & \text { if } x, y, z \in A(\rho) \\ -3 B(x y, z) n_{\rho} & \text { if } x, y, z \in A(-\rho)\end{cases}
$$

Using (6.3) and (6.4) we have 


$$
J(x, y, z)=3(x y) z \quad \text { for } x, y, z \in A(\sigma), \sigma= \pm \rho
$$

$$
(x y) z+x(y z)=0 \quad \text { for } x, y, z \in A(\sigma), \sigma= \pm \rho
$$

$$
(x y) x=0 \quad \text { for } x, y \in A(\sigma), \sigma= \pm \rho
$$

For the proof of $(6.8)$ we set $\sigma \equiv \sigma\left(n_{\rho}\right)$ and note that $-\sigma J(x, y, z)$ $=J\left(x, y, z n_{\rho}\right)=J\left(z n_{\rho}, x, y\right)$ and $J\left(x y \cdot z, n_{\rho}\right)=(x y, z) n_{\rho}+\left(z n_{\rho}\right)(x y)+\left(n_{\rho} \cdot x y\right) z$ $=\sigma x y \cdot z-\sigma z \cdot x y+\sigma x y \cdot z=3 \sigma x y \cdot z$. Therefore

$$
\begin{aligned}
3 \sigma x y \cdot z-\sigma J(x, y, z) & =J\left(z n_{\rho}, x, y\right)+J\left(x y, z, n_{\rho}\right) \\
& =J\left(z x, y, n_{\rho}\right)+J\left(y n_{\rho}, z, x\right) \\
& =\sigma J(y, z, x)
\end{aligned}
$$

which completes the proof.

Next let $w, x, y, z \in A(\sigma), \sigma= \pm \rho$, then

$$
\begin{aligned}
w J(x, y, z) & -x J(y, z, w)+y J(z, w, x)-z J(w, x, y) \\
& =3 / 2[2 J(w x, y, z)+2 J(y z, w, x)], \text { using }(3.5) \\
& =3 / 2[2 J(y, z, w x)+2 J(w, x, y z)] \\
& =3 / 2[3 y z \cdot w x+3 w x \cdot y z], \text { using }(6.8) \\
& =0 .
\end{aligned}
$$

This proves the important indentity

$$
\begin{array}{r}
w J(x, y, z)=x J(y, z, w)-y J(z, w, x)+z J(w, x, y) \text { for all } \\
w, x, y, z \in A(\sigma), \sigma= \pm \rho .
\end{array}
$$

THEOREM 6.10. If $\sigma= \pm \rho$, then the dimension of $A(\sigma)$ is one or three.

Proof. If the dimension of $A(\sigma)$ is $>1$, then there exist linearly independent elements $x_{i}$ and $x_{j}$ in $A(\sigma)$. Let $y_{k}$ denote an element in the corresponding dual basis for $A(-\sigma)$, as in the first part of this section. From (6.8), $3\left(x_{i} x_{j}\right) y_{j}=2 J\left(x_{i}, x_{j}, y_{j}\right)=2\left[\left(x_{i} x_{j}\right) y_{j}+\left(x_{j} y_{j}\right) x_{i}+\right.$ $\left.\left(y_{j} x_{i}\right) x_{j}\right]=2\left[\left(x_{i} x_{j}\right) y_{j}-n_{\rho} x_{i}\right]$ and therefore

$$
\begin{aligned}
\left(x_{\imath} x_{j}\right) y_{j} & =-2 n_{\rho} x_{i} \\
& =2 \sigma x_{i} .
\end{aligned}
$$

This last equation shows that $x_{i} x_{j} \neq 0$. Now $0 \neq y=x_{i} x_{j} \in A(-\sigma)$ and therefore there exists $x_{k} \in A(\sigma)$ so that $y x_{k} \neq 0$; for, otherwise $y A(\sigma)=0$ and from this we obtain $(y, A(\sigma))=0$ and so $y=0$. From (6.7) we see that $x_{k} \neq x_{i}$ or $x_{k} \neq x_{j}$ and so we have three distinct elements $x_{i}, x_{j}, x_{k} \in A(\sigma)$ such that, using (6.5) and (6.4), $0 \neq 3\left(x_{i} x_{j}\right) x_{k}=J\left(x_{i}, x_{j}, x_{k}\right)=(3 / \rho) B\left(x_{\imath} x_{j}, x_{k} n_{\rho}\right) n_{\rho}$. Now for any $w \in A(\sigma)$ we have 


$$
\begin{aligned}
(3 \sigma / \rho) B\left(x_{i} x_{j}, x_{k} n_{\rho}\right) w & =(3 / \rho) B\left(x_{i} x_{j}, x_{k} n_{\rho}\right) w n_{\rho} \\
& =w\left[(3 / \rho) B\left(x_{i} x_{j}, x_{k} n_{\rho}\right) n_{\rho}\right] \\
& =w J\left(x_{i}, x_{j}, x_{k}\right) \\
& =x_{i} J\left(x_{j}, x_{k}, w\right)-x_{j} J\left(x_{k}, w, x_{i}\right)+x_{k} J\left(w, x_{i}, x_{j}\right)
\end{aligned}
$$

using (6.9). Thus by (6.4) and the action of $n_{\mathrm{\rho}} F$ and $A(\sigma)$ we may conclude that the dimension of $A(\sigma)$ is 3 provided $x_{i}, x_{j}$ and $x_{k}$ are linearly independent. This result is clear by the choice of $x_{i}, x_{j}$ and $x_{k}$ and the fact $\left(x_{i} x_{j}\right) x_{k} \neq 0$.

Now if the dimension of $A(\rho)$ is 1 , we see that the weight space subalgebra $B(\rho)$ equals $x_{\rho} F \oplus n_{\rho} F \oplus x_{-\rho} F$ and is the usual split three dimensional Lie algebra. In the other case when the dimension of $A(\rho)$ is 3 , rewrite $\left\{x_{i}, x_{j}, x_{k}\right\}$ as $\left\{x_{1}, x_{2}, x_{3}\right\}$ and letting $\left\{y_{1}, y_{2}, y_{3}\right\}$ be the corresponding dual basis for $A(-\rho)$ we have $B(\rho)=A(-\rho) \oplus n_{\mathrm{\rho}} F \oplus$ $A(\rho)$ where

$$
\begin{aligned}
A(\rho) & =\left\{x_{1}, x_{2}, x_{3}\right\}, & A(-\rho) & =\left\{y_{1}, y_{2}, y_{3}\right\} \text { and } \\
x_{i} n_{\rho} & =\rho x_{i}, & y_{i} n_{\rho} & =-\rho y_{i}, \\
y_{i} x_{j} & =\delta_{i j} u_{\rho} & \text { for } i, j & =1,2,3 ; \\
A^{2}(\rho) & \subset A(-\rho) & \text { and } A^{2}(-\rho) & \subset A(\rho) .
\end{aligned}
$$

Next let $x_{i}, x_{j}$ be as above and write $x_{i} x_{j}=\sum_{n=1}^{3} a(i, j, n) y_{n}$, then for any other $x_{k}$ we have

$$
\left(x_{i} x_{j}, x_{k}\right)=\Sigma_{n} a(i, j, n)\left(y_{n}, x_{k}\right)=a(i, j, k) .
$$

This formula implies $a(i, j, k)$ is a skew-symmetric function for $i, j, k=1,2,3$. Thus since $a(i, j, i)=a(i, j, j)=0$ we have

$$
x_{i} x_{j}=a(i, j, k) y_{k} \text { for } k \neq i \text { or } j \text { and } i, j, k=1,2,3 \text {. }
$$

Thus setting $a=a(1,2,3) \in F$, we have

$$
x_{1} x_{2}=a y_{3}, \quad x_{2} x_{3}=a y_{1}, \quad x_{3} x_{1}=a y_{2} .
$$

Similarly if we set $y_{i} y_{j}=\sum_{n=1}^{3} b(i, j, n) x_{n}$ and $b(1,2,3)=b \in F$ we see $y_{1} y_{2}=b x_{3}, y_{2} y_{3}=b x_{1}$ and $y_{3} y_{1}=b x_{2}$. This gives the following table for multiplication in $B(\rho)$

\begin{tabular}{c|ccccccc} 
& $n_{\rho}$ & $x_{1}$ & $x_{2}$ & $x_{3}$ & $y_{1}$ & $y_{2}$ & $y_{3}$ \\
\hline$n_{\rho}$ & 0 & $-\rho x_{1}$ & $-\rho x_{2}$ & $-\rho x_{3}$ & $\rho y_{1}$ & $\rho y_{2}$ & $\rho y_{3}$ \\
$x_{1}$ & & 0 & $a y_{3}$ & $-a y_{2}$ & $n_{\rho}$ & 0 & 0 \\
$x_{2}$ & & & 0 & $a y_{1}$ & 0 & $n_{\rho}$ & 0 \\
$x_{3}$ & & & & 0 & 0 & 0 & $n_{\rho}$ \\
$y_{1}$ & & & & & 0 & $b x_{3}$ & $-b x_{2}$ \\
$y_{2}$ & & & & & & 0 & $b x_{1}$ \\
$y_{3}$ & & & & & & & 0
\end{tabular}


Now $a$ and $b$ are nonzero, otherwise $B(\rho)$ would not be simple and from (6.11) we see $2 \rho x_{1}=\left(x_{1} x_{2}\right) y_{2}=a y_{3} y_{2}=-a b x_{1}$ and therefore $2 \rho=-a b$.

Next we consider Malcev algebras [2]. This is an algebra which satisfies the identities obtained by introducing commutation $x \circ y=x y-y x$ as a new multiplicative operation in an alternative algebra. In particular if this is done in the split Cayley-Dickson algebra $C$, then we obtain an eight dimensional anti-commutative Malcev algebra $C^{-}$. In this algebra the identity 1 of $C$ is such that $1 \circ x=0$ for all $x \in C$ and so we set $C^{0}=C^{-} / 1 F$. It can be shown that $C^{0}$ is a simple Malcev algebra which satisfies the identities

$$
x y=-y x \text { and } J(x, y, x z)=J(x, y, z) x,
$$

omitting "o" as the notation for multiplication. Furthermore $C^{0}$ has a basis $\left\{u, e_{1}, e_{2}, e_{3}, e_{1}^{\prime}, e_{2}^{\prime}, e_{3}^{\prime}\right\}$ which satisfies the relations

$$
\begin{array}{lll}
u e_{i}=2 e_{i}, & u e_{i}^{\prime}=-2 e_{i}^{\prime}, & e_{i} e_{j}^{\prime}=\delta_{i j} u ; \\
e_{1} e_{2}=2 e_{3}^{\prime}, & e_{1} e_{3}=-2 e_{2}^{\prime}, & e_{2} e_{3}=2 e_{1}^{\prime} ; \\
e_{1}^{\prime} e_{2}^{\prime}=-2 e_{3}, & e_{1}^{\prime} e_{3}^{\prime}=2 e_{2}, & e_{2}^{\prime} e_{3}^{\prime}=-2 e_{1} .
\end{array}
$$

We now have

THEOREM 6.12. The root algebra $B(\rho)$ is a seven dimensional Malcev algebra as described above.

Proof. Since $F$ is algebraically closed, we can find $\lambda, \mu, v$ in $F$ such that $\lambda \rho=-2,-\lambda=\mu v, a \mu^{2}=2 v$ and $b v^{2}=-2 \mu$. Then make the following change of basis in $B(\rho)$ :

$$
H=\lambda n_{\rho}, \quad X_{i}=\mu x_{i}, \quad Y_{i}=v y_{i} \quad i=1,2,3
$$

and use the multiplication table for the $n_{\rho}, x_{i}, y_{i}$ to see that the $H, X_{i}, Y_{i}$ satisfy the above relations for a Malcev algebra with the identification $u=H, e_{i}=X_{i}, e_{i}^{\prime}=Y_{i}$.

We next show that the Lie algebra of linear transformations $R(N)$ can be simultaneously diagonalized in $A=\Sigma \rho A(-\rho) \oplus N \oplus \Sigma A(\rho)$, where the Cartan subalgebra $N$ equals $\Sigma_{\rho} A(-\rho) A(\rho)$. From the preceding discussion we know the dimension of $A(\rho)$ is one or three. Now in either case $A(\rho) A(-\rho)=n_{\rho} F$ and therefore $N$ is spanned by such elements $n_{\rho}$ for $\rho \neq 0$. Thus to show $R(N)$ diagonalizable it suffices to show all the linear transformations $R\left(n_{\sigma}\right)$ have this property on each subalgebra $B(\rho)$.

If the dimension of $A(\rho)$ is one, then this is clear for each $R\left(n_{\sigma}\right)$, where $\sigma$ is any nonzero weight. If the dimension of $A(\rho)$ is 3 , then 
$A(\rho)=\left\{x_{1}, x_{2}, x_{3}\right\}$ and $A(-\rho)=\left\{y_{1}, y_{2}, y_{3}\right\}$ where $\left\{y_{1}, y_{2}, y_{3}\right\}$ is a dual basis of $\left\{x_{1}, x_{2}, x_{3}\right\}$ and $\left\{x_{1}, x_{2}, x_{3}\right\}$ is a basis which simultaneously triangulates every $R\left(n_{\sigma}\right)$. We now show $\left\{y_{1}, y_{2}, y_{3}\right\}$ has the same property. For some $a_{i j} \in F$,

$$
\begin{aligned}
& x_{1} R\left(n_{\sigma}\right)=\rho\left(n_{\sigma}\right) x_{1} \\
& x_{2} R\left(n_{\sigma}\right)=a_{21} x_{1}+\rho\left(n_{\sigma}\right) x_{2} \\
& x_{3} R\left(n_{\sigma}\right)=a_{31} x_{1}+a_{32} x_{2}+\rho\left(n_{\sigma}\right) x_{3} .
\end{aligned}
$$

We shall show that the $a_{i j}=0$. Using the properties of $B(x, y)$ and the fact that $J\left(x_{2}, n_{\sigma}, y_{1}\right)=0$ we have

$$
\begin{aligned}
0 & =B\left(J\left(x_{2}, n_{\sigma}, y_{1}\right), n_{\rho}\right) \\
& =B\left(\left(x_{2} n_{\sigma}\right) y_{1}+\left(n_{\sigma} y_{1}\right) x_{2}+\left(y_{1} x_{2}\right) n_{\sigma}, n_{\rho}\right) \\
& =B\left(\left(x_{2} n_{\sigma}\right) y_{1}, n_{\rho}\right)+B\left(\left(n_{\sigma} y_{1}\right) x_{2}, n_{\rho}\right), \text { since } y_{1} x_{2}=0 \\
& =B\left(\left(x_{2} n_{\sigma}\right) y_{1}, n_{\rho}\right)+\rho B\left(n_{\sigma} y_{1}, x_{2}\right), \text { since } x_{i} n_{\rho}=\rho x_{i} \\
& =B\left(\left(x_{2} n_{\sigma}\right) y_{1}, n_{\rho}\right)
\end{aligned}
$$

using

$$
\begin{aligned}
B\left(n_{\sigma} y_{1}, x_{2}\right) & =\operatorname{trace} \bar{R}\left(n_{\sigma} y_{1}\right) \bar{R}\left(x_{2}\right)-\operatorname{trace} \bar{R}\left(n_{\sigma}\right) \bar{R}\left(y_{1} x_{2}\right) \\
& =\operatorname{trace} \bar{R}\left(n_{\sigma} y_{1} \cdot x_{2}+n_{\sigma} \cdot y_{1} x_{2}\right)=0
\end{aligned}
$$

since $n_{\sigma} y_{1} \cdot x_{2}+n_{\sigma} \cdot y_{1} x_{2} \in n_{\rho} F$ and trace $\bar{R}\left(n_{\rho}\right)=0$. Therefore since $\left(x_{2} n_{\sigma}\right) y_{1} \in n_{\rho} F$, we must have $\left(x_{2} n_{\sigma}\right) y_{1}=0$, since $B\left(n_{\rho}, n_{\rho}\right) \neq 0$. Now multiply $(6.14)$ by $y_{1}$ to obtain $0=\left(x_{2} n_{\sigma}\right) y_{1}=a_{21} x_{1} y_{1}+\rho\left(n_{\sigma}\right) x_{2} y_{1}$ $=-a_{21} n_{\rho}$ and therefore $a_{21}=0$. Similarly we can show $\left(x_{3} n_{\sigma}\right) y_{1}=\left(x_{3} n_{\sigma}\right) y_{2}$ $=0$ and conclude $a_{31}=a_{32}=0$ so that $R\left(n_{\sigma}\right)$ acts diagonally on $A(\rho)$.

Next suppose $y_{i} R\left(n_{\sigma}\right)=b_{i 1} y_{1}+b_{i 2} y_{2}+b_{i 3} y_{3}$ for $i=1,2,3$, then as before we can show $\left(y_{1} n_{\sigma}\right) x_{2}=\left(y_{1} n_{\sigma}\right) x_{3}=0$ and conclude that $b_{12}=$ $b_{13}=0$. Similarly for $i=2,3$ to obtain that $R\left(n_{\sigma}\right)$ acts diagonally on $A(-\rho)$. Thus for any weights $\rho, \sigma \neq 0, R\left(n_{\sigma}\right)$ acts diagonally on $B(\rho)$. But since $N=\Sigma_{\sigma} n_{\sigma} F$ and $A=\Sigma_{\rho} A(-\rho) \oplus N \oplus \Sigma_{\rho} A(\rho)=\Sigma_{\rho} B(\rho)$ we have

THEOREM 6.16. For any $n \in N, R(n)$ acts diagonally in $A$.

We shall now prove the theorem stated in the introduction. Let $\sigma \neq 0$ and $\rho \neq 0, \pm \sigma$ and let $x \in A(\rho), y \in A(\sigma)$ and suppose $0 \neq x y \epsilon$ $A(\rho) A(\sigma) \subset A(\rho+\sigma)$ so that $\rho+\sigma$ is a weight. Let $z \in A(\rho+\sigma)$, then for any $n \in N$ we have

$$
\begin{aligned}
J(x y, z, n) & =J(x y, z, n)+J(z n, x, y) \\
& =J(x z, n, y)+J(n y, x, z) \\
& =0
\end{aligned}
$$


using (3.15) for the first and third equalities. Since $z, x y \in A(\rho+\sigma)$ and $J(x y, z, n)=0$ we can conclude $x y \cdot z \in A(2(\rho+\sigma))=0$, using Theorem 5.13. This proves

$$
A(\rho) A(\sigma) \cdot A(\rho+\sigma)=0 \quad \text { if } \sigma \neq 0 \text { and } \rho \neq 0, \pm \sigma \text {. }
$$

Next we show

Lemma 6.18. The dimension of $A(\rho+\sigma)$ is one if $\sigma \neq 0$ and $\rho \neq 0, \pm \sigma$ and $A(\rho) A(\sigma) \neq 0$.

Proof. Suppose dimension $A(\rho+\sigma)=3$, then form $B(\rho+\sigma)$ $=A(-(\rho+\sigma)) \oplus n_{\rho+\sigma} F \oplus A(\rho+\sigma)$. From the hypothesis we can find an element $z=x y \in A(\rho) A(\sigma) \subset A(\rho+\sigma)$ which is not zero and such that $z A(\rho+\sigma) \neq 0$; this last statement follows from the multiplicative relations for the Malcev algebra $B(\rho+\sigma)$; (briefly: otherwise, $\left.B(z, A(-(\rho+\sigma)))=B\left(z, A(\rho+\sigma)^{2}\right)=B(z A(\rho+\sigma), A(\rho+\sigma))=0\right)$. But the fact $z A(\rho+\sigma) \neq 0$ contradicts (6.17).

Now let $G(\sigma)$ denote a weight space of dimension one and $S(\sigma)$ denote a weight space of dimension three and set

$$
\begin{aligned}
& G=\Sigma_{\sigma}\left(G(-\sigma) \oplus n_{\sigma} F \oplus G(\sigma)\right) \\
& S=\Sigma_{\rho}\left(S(-\rho) \oplus n_{\rho} F \oplus S(\rho)\right) .
\end{aligned}
$$

Then $A=G+S$ and we shall show $G$ is an ideal in $A$. For any weight $\rho$ with weight space $G(\rho)$ we have $G G(\rho) \subset G$, since the product of two weight spaces of dimension one is at most of dimension one. Next if $\lambda$ is a weight with weight space $S(\lambda)$ of dimension 3 , then $G(\rho) S(\lambda) \subset G(\rho+\lambda)$ by Lemma 6.18. Now if $n_{\sigma} \in G$, then write $n_{\sigma}=y x$ where $y \in G(-\sigma), x \in G(\sigma)$. If $z \in S(\lambda)$ (where we know $\lambda \neq \pm \sigma)$, we have $0=J(y, x, z)=y x \cdot z+x z \cdot y+z y \cdot x$ and therefore

$$
\begin{aligned}
n_{\sigma} z=y x \cdot z= & -x z \cdot y \\
& -z y \cdot x \in G(\sigma) S(\lambda) \cdot G(-\sigma)+G(-\sigma) S(\lambda) \cdot G(\sigma) \subset G .
\end{aligned}
$$

Thus $G S(\lambda) \subset G$. Since $G N \subset G$, we combine the above results to see that $G$ is an ideal of $A$. But since $A$ is simple $G=0$ or $G=A$; in the latter case $A$ is a Lie algebra.

So next suppose $G=0$ and let $\rho \neq 0$ be a fixed weight and $\sigma \neq 0$, $\pm \rho$ any other weight and form $B(\rho)=A(-\rho) \oplus n_{\rho} F \oplus A(\rho)$.

Case 1. $A(\rho) A(\sigma)=0$. Then $B(\rho) A(\sigma)=A(-\rho) A(\sigma)+n_{\rho} A(\sigma)$. Now if $A(-\rho) A(\sigma)=0$ also, then $n_{\mathrm{\rho}} A(\sigma)=0$. For let $x \in A(\rho), \mathrm{y} \in A(-\rho)$ be such that $x y=n_{\rho}$ and let $z \in A(\sigma)$, then 


$$
0=J(x, y, z)=x y \cdot z+y z \cdot x+z x \cdot y=x y \cdot z=n_{\rho} z
$$

and we would have $B(\rho) A(\sigma)=0$. Thus we want to show $A(-\rho) A(\sigma)=0$, so suppose this is not true. Then $0 \neq A(-\rho) A(\sigma) \subset A(\sigma-\rho)$ and from Lemma 6.18 the dimension of $A(\sigma-\rho)$ is one and therefore $A(\sigma-\rho) \subset G=0$. Thus we may actually conclude $A(-\rho) A(\sigma)=0$ and this proves

$$
B(\rho) A(\sigma)=0 \quad \text { if } A(\rho) A(\sigma)=0 .
$$

Case 2. $A(\rho) A(\sigma) \neq 0$. Then $0 \neq A(\rho) A(\sigma) \subset A(\rho+\sigma)$ and again by Lemma 6.18 the dimension of $A(\rho+\sigma)$ is one. Thus, as above, this yields $A(\rho) A(\sigma)=0$, a contradiction. Thus we may conclude from these cases that $B(\rho) A(\sigma)=0$ if $\rho \neq 0, \sigma \neq 0, \pm \rho$. This yields $B(\rho) A \subset$ $B(\rho)$ which means $B(\rho)$ is a nonzero ideal of $A$ and so $A=B(\rho)$.

\section{REFERENCES}

1. N. Jacobson, Lie Algebras, Interscience, 1962.

2. A. Sagle, On simple Malcev algebras over fields of characteristic zero, Pacific J. Math. 12 (1962), 1057-1078.

3. A. Sagle, On anti-commutative algebras and analytic loops, to appear in Canad. J. Math.

University of CALIFornia, Los ANGELes 


\title{
PACIFIC JOURNAL OF MATHEMATICS
}

\author{
EDITORS
}

\author{
H. Samelson \\ Stanford University \\ Stanford, California \\ R. M. Blumenthal \\ University of Washington \\ Seattle, Washington 98105
}

\author{
J. DugundjI \\ University of Southern California \\ Los Angeles, California 90007 \\ Richard Arens \\ University of California \\ Los Angeles, California 90024
}

\section{ASSOCIATE EDITORS}
E. F. BECKENBACH
B. H. NEUMaNN
F. WOLF
K. YosIDA

\section{SUPPORTING INSTITUTIONS}

\author{
UNIVERSITY OF BRITISH COLUMBIA \\ CALIFORNIA INSTITUTE OF TECHNOLOGY \\ UNIVERSITY OF CALIFORNIA \\ MONTANA STATE UNIVERSITY \\ UNIVERSITY OF NEVADA \\ NEW MEXICO STATE UNIVERSITY \\ OREGON STATE UNIVERSITY \\ UNIVERSITY OF OREGON \\ OSAKA UNIVERSITY \\ UNIVERSITY OF SOUTHERN CALIFORNIA
}

\author{
STANFORD UNIVERSITY \\ UNIVERSITY OF TOKYO \\ UNIVERSITY OF UTAH \\ WASHINGTON STATE UNIVERSITY \\ UNIVERSITY OF WASHINGTON \\ AMERICAN MATHEMATICAL SOCIETY \\ CALIFORNIA RESEARCH CORPORATION \\ SPACE TECHNOLOGY LABORATORIES \\ NAVAL ORDNANCE TEST STATION
}

Mathematical papers intended for publication in the Pacific Journal of Mathematics should by typewritten (double spaced). The first paragraph or two must be capable of being used separately as a synopsis of the entire paper. It should not contain references to the bibliography. Manuscripts may be sent to any one of the four editors. All other communications to the editors should be addressed to the managing editor, Richard Arens, at the University of California, Los Angeles, California 90024.

50 reprints per author of each article are furnished free of charge; additional copies may be obtained at cost in multiples of 50 .

The Pacific Journal of Mathematics is published quarterly, in March, June, September, and December. Effective with Volume 13 the price per volume (4 numbers) is $\$ 18.00$; single issues, $\$ 5.00$. Special price for current issues to individual faculty members of supporting institutions and to individual members of the American Mathematical Society: $\$ 8.00$ per volume; single issues $\$ 2.50$. Back numbers are available.

Subscriptions, orders for back numbers, and changes of address should be sent to Pacific Journal of Mathematics, 103 Highland Boulevard, Berkeley 8, California.

Printed at Kokusai Bunken Insatsusha (International Academic Printing Co., Ltd.), No. 6, 2-chome, Fujimi-cho, Chiyoda-ku, Tokyo, Japan.

PUBLISHED BY PACIFIC JOURNAL OF MATHEMATICS, A NON-PROFIT CORPORATION

The Supporting Institutions listed above contribute to the cost of publication of this Journal, but they are not owners or publishers and have no responsibility for its content or policies. 


\section{Pacific Journal of Mathematics}

\section{Vol. 15, No. $2 \quad$ October, 1965}

Patrick Robert Ahern, On the generalized F. and M. Riesz theorem......... 373

A. A. Albert, On exceptional Jordan division algebras ................ 377

J. A. Anderson and G. H. Fullerton, On a class of Cauchy exponential

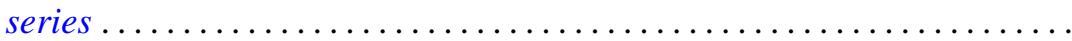

Allan Clark, Hopf algebras over Dedekind domains and torsion in

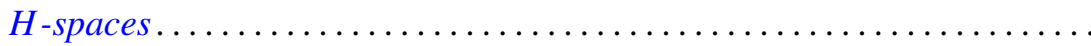

John Dauns and D. V. Widder, Convolution transforms whose inversion functions have complex roots .............................

Ronald George Douglas, Contractive projections on an $\mathrm{L}_{1}$ space ..........

Robert E. Edwards, Changing signs of Fourier coefficients ...............

Ramesh Anand Gangolli, Sample functions of certain differential processes on symmetric spaces .....................................

Robert William Gilmer, Jr., Some containment relations between classes of

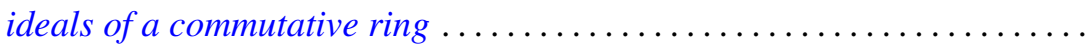

Basil Gordon, A generalization of the coset decomposition of a finite

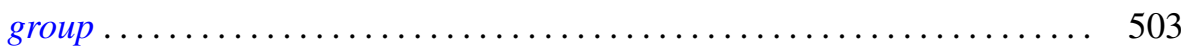

Teruo Ikebe, On the phase-shift formula for the scattering operator....... 511

Makoto Ishida, On algebraic homogeneous spaces ................ 525

Donald William Kahn, Maps which induce the zero map on homotopy ........ 537

Frank James Kosier, Certain algebras of degree one ................. 541

Betty Kvarda, An inequality for the number of elements in a sum of two sets of lattice points.................................

Jonah Mann and Donald J. Newman, The generalized Gibbs phenomenon for regular Hausdorff means. .

Charles Alan McCarthy, The nilpotent part of a spectral operator. II . ...

Donald Steven Passman, Isomorphic groups and group rings ...

R. N. Pederson, Laplace's method for two parameters .....

Tom Stephen Pitcher, A more general property than domination for sets of probability measures .............................

Arthur Argyle Sagle, Remarks on simple extended Lie algebras. .

Arthur Argyle Sagle, On simple extended Lie algebras over fields of

characteristic zero.

Tôru Saitô, Proper ordered inverse semigroups ...........

Oved Shisha, Monotone approximation

Indranand Sinha, Reduction of sets of matrices to a triangular form

Raymond Earl Smithson, Some general properties of multi-valued

functions .................................

John Stuelpnagel, Euclidean fiberings of solvmanifolds .... 